\title{
Investigating early-type galaxy evolution with a multiwavelength approach
}

\section{The UV structure of 11 galaxies with Swift-UVOT ${ }^{\star}$}

\author{
R. Rampazzo ${ }^{1}$, P. Mazzei ${ }^{1}$, A. Marino ${ }^{1}$, M. Uslenghi ${ }^{2}$, G. Trinchieri $^{3}$, and A. Wolter ${ }^{3}$ \\ 1 INAF Osservatorio Astronomico di Padova, Vicolo dell'Osservatorio 5, 35122 Padova, Italy \\ e-mail: roberto.rampazzo@oapd.inaf.it \\ 2 INAF-IASF, via E. Bassini 15, 20133 Milano, Italy \\ 3 INAF Osservatorio Astronomico di Brera, via Brera 28, 20121 Milano, Italy
}

Received 19 September 2016 / Accepted 3 February 2017

\begin{abstract}
Context. GALEX detected a significant fraction of early-type galaxies, in particular S0s, showing far-UV bright structures, sometimes involving an entire galaxy out to its outskirts. These features suggest the presence of either recent ongoing and/or prolonged star formation episodes, shedding new light on the evolution of these systems.

Aims. We aim at understanding the evolutionary path[s] of these early-type galaxies and the mechanisms at the origin of their UVbright structures. We investigate with a multiwavelength approach the link between the inner and outer galaxy regions of a set of 11 early-type galaxies that were selected because of their nearly passive stage of evolution in the nuclear region.

Methods. This paper, second of a series, focuses on the information coming from the comparison between UV features detected by Swift-UVOT, which trace recent star formation, and the galaxy optical structure, which maps older stellar populations. We performed a surface photometric study of these early-type galaxies, observed with the Swift-UVOT UV filters W2 $2030 \AA \lambda_{0}, M 22231 \AA \lambda_{0}$, $W 12634 \AA \lambda_{0}$ and the $U B V$ bands. BVRI photometry from other sources in the literature was also used. Our integrated magnitude measurements were analyzed and compared with corresponding values in the literature. We characterize the overall galaxy structure that best fits the UV and optical luminosity profiles using a single Sérsic law.

Results. The galaxies NGC 1366, NGC 1426, NGC 3818, NGC 3962, and NGC 7192 show featureless luminosity profiles. Excluding NGC 1366, which has a clear edge-on disk $(n \approx 1-2)$, and NGC 3818, the remaining three galaxies have Sérsic's indices $n \approx 3-4$ in the optical and a lower index in the UV. Bright ring- or arm-like structures are revealed by UV images and luminosity profiles of NGC 1415, NGC 1533, NGC 1543, NGC 2685, NGC 2974, and IC 2006. The ring- or arm-like structures differ from galaxy to galaxy. Sérsic indices of UV profiles for these galaxies are in the range $n=1.5-3$ both in S0s and in galaxies classified as bona fide ellipticals, such as NGC 2974 and IC 2006. We note that in our sample optical Sérsic indices are usually higher than in the UV indices. $(M 2-V)$ color profiles are bluer in ring- or arm-like structures than in the galaxy body.

Conclusions. The lower values of Sérsic indices in the UV bands with respect to optical bands, suggesting the presence of a disk, point out that the role of the dissipation cannot be neglected in recent evolutionary phases of these early-type galaxies.
\end{abstract}

Key words. galaxies: elliptical and lenticular, $\mathrm{cD}$ - galaxies: fundamental parameters - ultraviolet: galaxies - galaxies: evolution

\section{Introduction}

The combination of the far-UV view provided by GAlaxy Evolution EXplorer (GALEX; Martin et al. 2005) with the Sloan Digital Sky Survey (SDSS; Stoughton et al. 2002) has greatly undermined the classical view of early-type galaxies (Es + S0s = ETGs hereafter) as passively evolving galaxies. Statistical studies (e.g., Kaviraj et al. 2007; Schawinski et al. 2007) found that about $30 \%$ of massive ETGs show recent or ongoing star formation, with the largest incidence in galaxies located in low-density environments. Only a small percentage of cluster ETGs, 5/9\%, shows the classical UV upturn that is due to evolved risingbranch stars, whereas $\sim 27 / 43 \%$ shows recent star formation (Yi et al. 2011; Hernández-Pérez \& Bruzual 2014, respectively).

* Full Table A.1 is only available at the CDS via anonymous ftp to cdsarc.u-strasbg.fr (130.79.128.5) or via

http://cdsarc.u-strasbg.fr/viz-bin/qcat?]/A+A/602/A97
In color-magnitude diagrams (CMD) built with SDSS and GALEX magnitudes, galaxies in clusters show a strong morphological segregation: spirals lie in the blue cloud, while ETGs are mainly located in the red sequence (e.g., Yi et al. 2005; Wyder et al. 2007). However, an intermediate area, called green valley exists, regardless of the environment. Recently, Mazzei et al. (2014a) provided a description of the galaxy photometric and morphological evolution in the (NUV $-r$ ) vs. $M_{r}$ CMD plane by applying an SPH code with chemo-photometric implementation to ETGs in the Leo II cloud. Major merging as well as galaxy-galaxy interaction are the mechanisms required to match the global properties of such ETGs. The simulations show that these galaxies spend between 3 to $5 \mathrm{Gyr}$, depending on their luminosity, in crossing the green valley before they reach the red sequence. The simulations also show that rejuvenation episodes are more frequent in less massive ETGs.

GALEX revealed that ETGs with signatures of recent accretion episodes, like shell structures, may have a "rejuvenated" 
Table 1. General properties of the sample.

\begin{tabular}{llllllllll}
\hline \hline $\begin{array}{l}\text { Galaxy } \\
\text { ident. }\end{array}$ & $\begin{array}{l}\text { Morpho. } \\
\text { RSA }\end{array}$ & Type & $M_{K}$ & $\epsilon$ & $\begin{array}{l}\text { PA } \\
{[\mathrm{deg}]}\end{array}$ & $\begin{array}{l}D \\
{[\mathrm{Mpc}]}\end{array}$ & $\begin{array}{l}M(\mathrm{HI}) \\
10^{9} M_{\odot}\end{array}$ & $\begin{array}{l}\text { Group } \\
\text { name }\end{array}$ & $\begin{array}{l}\rho_{x y z} \\
{\left[\mathrm{gal} \mathrm{Mpc}^{-3}\right]}\end{array}$ \\
\hline NGC 1366 & $\mathrm{S0}_{1}(8)$ & $-2.3 \pm 0.7$ & -22.59 & 0.54 & 3.8 & $21.1 \pm 2.1$ & $<1.0$ & Fornax Eridanus Cloud & 0.16 \\
NGC 1415 & $\mathrm{Sa} / \mathrm{SBa}$ late & $0.5 \pm 1.3$ & -23.47 & 0.59 & 141.2 & $22.7 \pm 1.5$ & 0.9 & " & 0.80 \\
NGC 1426 & $\mathrm{E} 4$ & $-4.8 \pm 0.5$ & -23.22 & 0.32 & 112.5 & $24.1 \pm 2.4$ & $\ldots$ & " & 0.66 \\
NGC 1533 & SB0 2 (2)/SBa & $-2.5 \pm 0.6$ & -23.97 & 0.37 & 148.9 & $21.4 \pm 2.1$ & $7.4^{a}$ & Dorado cloud & 0.89 \\
NGC 1543 & $\mathrm{RSB0}_{2 / 3}(0) / \mathrm{a}$ & $-2.0 \pm 0.8$ & -24.04 & 0.77 & 93.8 & $20.0 \pm 2.0$ & 0.8 & " & 0.95 \\
NGC 2685 & $\mathrm{SO}_{3}(7)$ pec & $-1.0 \pm 0.8$ & -22.64 & 0.46 & 38.0 & 16.0 & $3.0^{b}$ & Ursa Major South Spur & 0.13 \\
NGC 2974 & E4 & $-4.2 \pm 1.2$ & -25.42 & 0.38 & 44.0 & $21.5 \pm 2.2$ & $0.7^{c}$ & Lynx Cloud & 0.26 \\
NGC 3818 & E5 & $-4.6 \pm 0.8$ & -23.94 & 0.37 & 95.9 & $36.3 \pm 3.6$ & $\ldots$ & Crater Cloud & 0.20 \\
NGC 3962 & E1 & $-4.8 \pm 0.4$ & -25.07 & 0.28 & 10.0 & $35.3 \pm 3.5$ & $2.8^{d}$ & " & 0.32 \\
NGC 7192 & $\mathrm{SO}_{2}(0)$ & $-3.9 \pm 0.7$ & -24.37 & 0.05 & 9.9 & $37.8 \pm 3.8$ & $<0.7^{d}$ & Pavo Indus Spur & 0.28 \\
IC 2006 & E1 & $-4.2 \pm 0.9$ & -23.04 & 0.17 & 34.8 & $20.2 \pm 2.0$ & 0.3 & Fornax Eridanus Cloud & 0.82 \\
\hline
\end{tabular}

Notes. The morphology (Col. 2) is from the Sandage \& Tammann (1987) catalog. The morphological type, $T$ (Col. 3), the ellipticity, $\epsilon$ (Col. 5), and the position angle, PA (Col. 6), are derived from Hyperleda (Makarov et al. 2014) except for NGC 7192, which is taken from the CGS atlas. The $K_{\mathrm{s}}$ absolute magnitude (Col. 4) is from 2MASS. The adopted distances (Col. 7) are derived from the Extragalactic Distance Database (Tully et al. 2009). The HI masses (Col. 8) are obtained using the distance in Col. 7 and fluxes from NED and the following references: ${ }^{(a)}$ Ryan-Weber et al. (2003); ${ }^{(b)}$ Józsa et al. (2009); ${ }^{(c)}$ Kim et al. (1988); ${ }^{(d)}$ Serra \& Oosterloo (2010). The group identification (Col. 9) and the local galaxy density, $\rho_{x y z}\left(\right.$ Col. 10), i.e., the density of galaxies brighter than $M_{B}=-16 \mathrm{mag}$, are adopted from Tully (1988).

nucleus in the far-UV (Rampazzo et al. 2007; Marino et al. 2009). This agrees with results from the study of Lick spectroscopic indices by Longhetti et al. (2000) and Annibali et al. (2007) concerning ages within $r_{\mathrm{e}} / 8$, that is, in the nuclear region, and more recently, from mid-infrared (MIR) Spitzer-IRS spectral analysis within $\approx 2-3 r_{\mathrm{e}} / 8$. This last finding highlights the role of polycyclic aromatic hydrocarbon features (PAHs) as tracers of episodes of star formation with ages in the range between 1 and $2.5 \mathrm{Gyr}$ in a large portion of ETGs, with the exact values depending on the metallicity. This look-back time corresponds to a redshift $z \lesssim 0.2$ (Bressan et al. 2006; Kaneda et al. 2008; Vega et al. 2010; Panuzzo et al. 2011; Rampazzo et al. 2013; Nanni et al. 2013).

Furthermore, ETGs can show far-UV bright extended structures (e.g., rings, arm-like features, tails), sometimes completely distinct in shape from the optical galaxy body, and often associated with HI emission (Jeong 2009; Thilker et al. 2010; Marino et al. 2011c,a; Salim \& Rich 2010; Rampazzo et al. 2011; Salim et al. 2012). Simulations suggest that such UV bright ring- or arm-like structures can be either a transient product of a major merger (Mazzei et al. 2014a) or can result from the accretion of a small gas-rich companion (Mapelli et al. 2015). Observations and simulations both indicate that galaxy-scale rejuvenation processes occur at least in some ETGs, most likely fed by either residual or freshly accreted gas.

This paper, the second of a series based on Swift (Gehrels et al. 2004; Citterio et al. 1994; Burrows et al. 2005) multiwavelength (XRT + UVOT) observations, is dedicated to tracing signatures of recent evolution in nearby ETGs. The sample only includes the 11 galaxies in Trinchieri et al. (2015, Paper I hereafter) observed by Swift. Here, we examine the near-UV (NUV) galaxy structures revealed by UVOT. Galaxies are selected based on the nearly passive stage of evolution of their nuclear region (Rampazzo et al. 2013). Most of these galaxies, which based on the spectrum of their nucleus may be considered as templates of nearly dead ETGs, reveal many bright and peculiar structures in our NUV Swift-UVOT images (Fig. 7 of Paper I). We investigate the information contained in the NUV about the history of these galaxies. We also explore whether a common framework may be found for all these sources. The comparison between the NUV, which traces recent episodes of star formation, and the optical spatial structures, which map older stellar populations, might provide indications about the formation mechanisms of the NUV-bright structures that link recent and past ETG evolutionary scenarios. The study of the galaxy structures has recently developed through the multiwavelength comparison of the Sérsic indices derived from the analysis of the luminosity profiles. Vulcani et al. (2014) fit a set of bands from $u$ to $H$ of a low-redshift galaxy sample taken from the Galaxy And Mass Assembly (GAMA) survey with a single Sérsic law. They examined both late-type galaxies and ETGs, that is, $n<2.5$ and $n>2.5$, in their definition. Late-types show an increase in Sérsic index with wavelength (see also Kennedy et al. 2016), which is different from ETGs, for which $n$ is almost constant (see also La Barbera et al. 2010). The authors concluded that the variation in Sérsic index with wavelength is connected to the presence of the disk, and it is also most likely connected to a radial variation in stellar populations and/or dust reddening. The NUV is so far unexplored in this analysis, and we aim to extend the investigation in this direction using our Swift-UVOT data set.

The paper plan is the following. In Sect. 2 we recall our sample, and in Sect. 3 we describe observations and the reduction techniques we adopted. Difficulties in an accurate surface brightness analysis of UVOT data are reviewed as well as the reduction packages. In Sect. 4 we present the results, which mainly consider the comparison between the NUV and the optical spatial light distribution. The discussion in the light of the literature is given in Sect. 5. Finally, Sect. 6 summarizes our results and conclusions.

\section{Sample}

Table 1 presents the general characteristics of our ETG sample. The table does not include NGC 1209, which was part of the investigation in Paper I, since the galaxy does not have Swift observations. All galaxies are ETGs according to their classification in Cols. 2 and 3. A transition case between early and later types is NGC 1415, whose classification has a large uncertainty, however. Their absolute $K_{\mathrm{s}}$ magnitude range (Col. 4), $-25.42 \leq M_{K_{\mathrm{s}}} \leq-22.59$, suggests a large interval in the stellar mass, and Col. 8 indicates that these ETGs are gas rich on average, with $\mathrm{HI}$ masses on the order of $10^{9} M_{\odot}$. Columns 9 
R. Rampazzo et al.: Investigating ETGs evolution with a multiwavelength approach. II.

and 10 refer to their environmental properties. The range of the local densities, $0.13 \leq \rho_{x y z}\left(\mathrm{gal} \mathrm{Mpc}^{-3}\right) \leq 0.95$ is large, but in general, they are in a relatively poor environment. Galaxies affiliated to the Fornax cluster and the Eridanus cloud are peripheral enough to be located in regions with densities typical of groups. The adopted distances from the Extragalactic Distance Database (Tully et al. 2009), quoted in Col. 7, have uncertainties of about $10 \%$.

The sample includes only galaxies that were selected because in their nuclear region, $2-3 \times r_{\mathrm{e}} / 8$, their MIR spectral classes range from 2 to 0 (Rampazzo et al. 2013, and references therein). MIR classes (Panuzzo et al. 2011) describe the spectral characteristics of the nuclear regions. The class 2 spectra show atomic and molecular emission lines plus PAHs features with anomalous interband ratios; the class 1 spectra show emission lines without PAHs, while class 0 spectra are characterized solely by the silicate emission at $\lambda \sim 10 \mu \mathrm{m}$ and the $\lambda \sim 18 \mu \mathrm{m}$ from evolved stars. Bressan et al. (2006) showed that class 0 spectra characterize passively evolving nuclei. The sample avoids class 3 spectra. In contrast to class 2, class 3 spectra show normal PAHs interband ratios that are typical of star-forming galaxies like spirals. The sample does not include class 4 spectra. These spectra differ from class 2 spectra because they are dominated by a hot dust continuum and sometimes show high ionization lines, which both indicate the presence of an AGN. To summarize, the class 2-1 nuclear spectra of our ETGs are consistent with traces of a past star formation and with possible residual AGN activity (see also Vega et al. 2010), while those of class 0 correspond to a passively evolving stellar population.

According to the galaxy morphology, which is presented for each band in Fig. 7 of Paper I, the sample can be divided into two sets. NGC 1366, NGC 1426, NGC 3818, NGC 3962, and NGC 7192 do not show remarkable features in either the optical or the NUV bands. The remaining six ETGs, namely NGC 1415, NGC 1533, NGC 1543, NGC 2685, NGC 2974, and NGC IC 2006, show bright sometimes peculiar ring- or arm-like features in the NUV filters.

\section{Observations and data reduction}

UVOT is a $30 \mathrm{~cm}$ telescope in the Swift platform operating both in imaging and spectroscopy modes (Roming et al. 2005). We observed our ETGs in imaging in all six available filters, $W 2$ ( $\lambda_{0}$ 2030), M2 ( $\lambda_{0}$ 2231), $W 1$ ( $\lambda_{0}$ 2634), $U$ ( $\lambda_{0}$ 3501), $B$ $\left(\lambda_{0} 4329\right), V\left(\lambda_{0} 5402\right)$. Description of the filters, PSFs (FWHM 2'.92 for $W 2,2$ ".45 for $M 2,2^{\prime \prime} .37$ for $W 1,2$ '!37 for $U, 2$ ".19 for $B, 2^{\prime \prime}$.18 for $\left.V\right)$, and calibrations are discussed in Breeveld et al. (2010, 2011).

UVOT data obtained in imaging mode with a $2 \times 2$ binning, resulting in $1.004^{\prime \prime} /$ pixel, were processed using the procedure described online ${ }^{1}$ We combined all the images taken in the same filter for each galaxy in a single image using UVOTSUM to improve the signal-to-noise ratio $(\mathrm{S} / \mathrm{N})$ and to enhance the visibility of NUV features of low surface brightness.

The final data set therefore contains $W 2, M 2, W 1, U, B$, and $V$ images for each galaxy observed with $S$ wift, as we discuss below. The final exposure times per image are different since we complied with the request of preserving the lifetime of the filter wheel and therefore observed as much as possible in the filterof-the-day. Total exposure times, previously given in Table 3 of Paper I, are reported in Table 2 for convenience.

\footnotetext{
1 http://WwW.swift.ac.uk/analysis/uvot/
}

Table 2. Total exposure times in the UVOT filters.

\begin{tabular}{lrrrrrr}
\hline \hline $\begin{array}{l}\text { Galaxy } \\
\text { Ident. }\end{array}$ & $\begin{array}{l}W 2 \\
{[\mathrm{~s}]}\end{array}$ & $\begin{array}{r}M 2 \\
{[\mathrm{~s}]}\end{array}$ & $\begin{array}{r}W 1 \\
{[\mathrm{~s}]}\end{array}$ & $\begin{array}{r}U \\
{[\mathrm{~s}]}\end{array}$ & $\begin{array}{r}B \\
{[\mathrm{~s}]}\end{array}$ & $\begin{array}{r}V \\
{[\mathrm{~s}]}\end{array}$ \\
\hline NGC 1366 & 13483 & 11825 & 3597 & 8627 & 1112 & 1112 \\
NGC 1415 & 23990 & 18595 & 22887 & 14857 & 1607 & 1562 \\
NGC 1426 & 9924 & 5744 & 11179 & 5765 & 544 & 544 \\
NGC 1533 & 7412 & 7220 & 5607 & 13198 & 1848 & 1848 \\
NGC 1543 & 17414 & 29743 & 20155 & 15133 & 1922 & 1575 \\
NGC 2685 & 7838 & 6517 & 6456 & 783 & 783 & 783 \\
NGC 2974 & 16495 & 6709 & 3548 & 7689 & 1082 & 1082 \\
NGC 3818 & 10616 & 8497 & 3296 & 15392 & 424 & 233 \\
NGC 3962 & 8487 & 11333 & 10490 & 6699 & 808 & 792 \\
NGC 7192 & 6927 & 15313 & 6163 & 6787 & 987 & 987 \\
IC 2006 & 12014 & 6238 & 4992 & 11181 & 1431 & 1338 \\
\hline
\end{tabular}

We used the photometric zero-points provided by Breeveld et al. (2011) to convert UVOT count rates into the AB magnitude system (Oke 1974): $z p_{W 2}=19.11 \pm 0.03, z p_{M 2}=18.54 \pm 0.03$, $z p_{W 1}=18.95 \pm 0.03, z p_{U}=19.36 \pm 0.02, z p_{B}=18.98 \pm 0.02$, and $z p_{V}=17.88 \pm 0.01$.

UVOT is a photon-counting instrument and is therefore subject to coincidence loss when the throughput is high, whether this is due to background or source counts, which may result in an undercounting of the flux. This effect is a function of brightness of the source and affects the linearity of the detector. The $U$, $B$, and $V$ filters are most affected, although coincidence loss can also be present in NUV filters. For our binning, Hoversten et al. (2011, and reference therein) calculates that count rates lower than 0.028 counts s$^{-1}$ pixel $^{-1}$ are affected by at most $1 \%$ due to coincidence loss. We checked for coincidence loss in all our images. As an example we show the count rates of NGC 1543 in the NUV and optical bands in Fig. 1. The NUV filters $U V W 2$, $U V M 2$, and $U V W 1$ are almost free of coincidence loss effects. We verified that in $U V W 1$, which is the most affected of the NUV filters, the region is restricted to a few pixels centered on the galaxy nucleus. For instance, in the case of NGC 1415, the level of the 0.028 counts $\mathrm{s}^{-1}$ pixel $^{-1}$ in the $U V W 1$ filter is exceeded in two pixels at the opposite sides of the innermost annulus revealed.

Coincidence loss effects can be corrected in the case of point sources (Poole et al. 2008; Breeveld et al. 2010). For extended sources a correction process has been performed for NGC 4449, a Magellanic-type irregular galaxy with bright star-forming regions, by Karczewski et al. (2013). Even though their whole field is affected, the authors calculated that the statistical and systematic uncertainties in their total fluxes amount to $\approx 7-9 \%$ overall for the NUV and the optical bands. Based on these results, we decided to refrain from applying any correction for coincidence loss to our optical data.

We considered the presence of instrumental scattered light, which in the NUV filters may cover the whole frame, and of the light scattered from stars (e.g., Hodges-Kluck \& Bregman 2014). This latter may produce ghost-images: for particularly bright stars, a ring pattern is produced. The frames most affected by these two effects are the $W 2$ and $W 1$ filters of NGC 1366 (see Sect. 4.4). Our images are the sum of several (dithered and rotated) frames. This sum tends to smooth out large-scale inhomogeneities in the final frame. Our targets, however, cover a limited portion of the $17^{\prime} \times 17^{\prime}$ of the UVOT field of view. The background, although not homogeneous because of the above factors, can be well evaluated around each object.

Surface photometry has been performed using the ELLIPSE (Jedrzejewski 1987) fitting routine in the STSDAS package of 


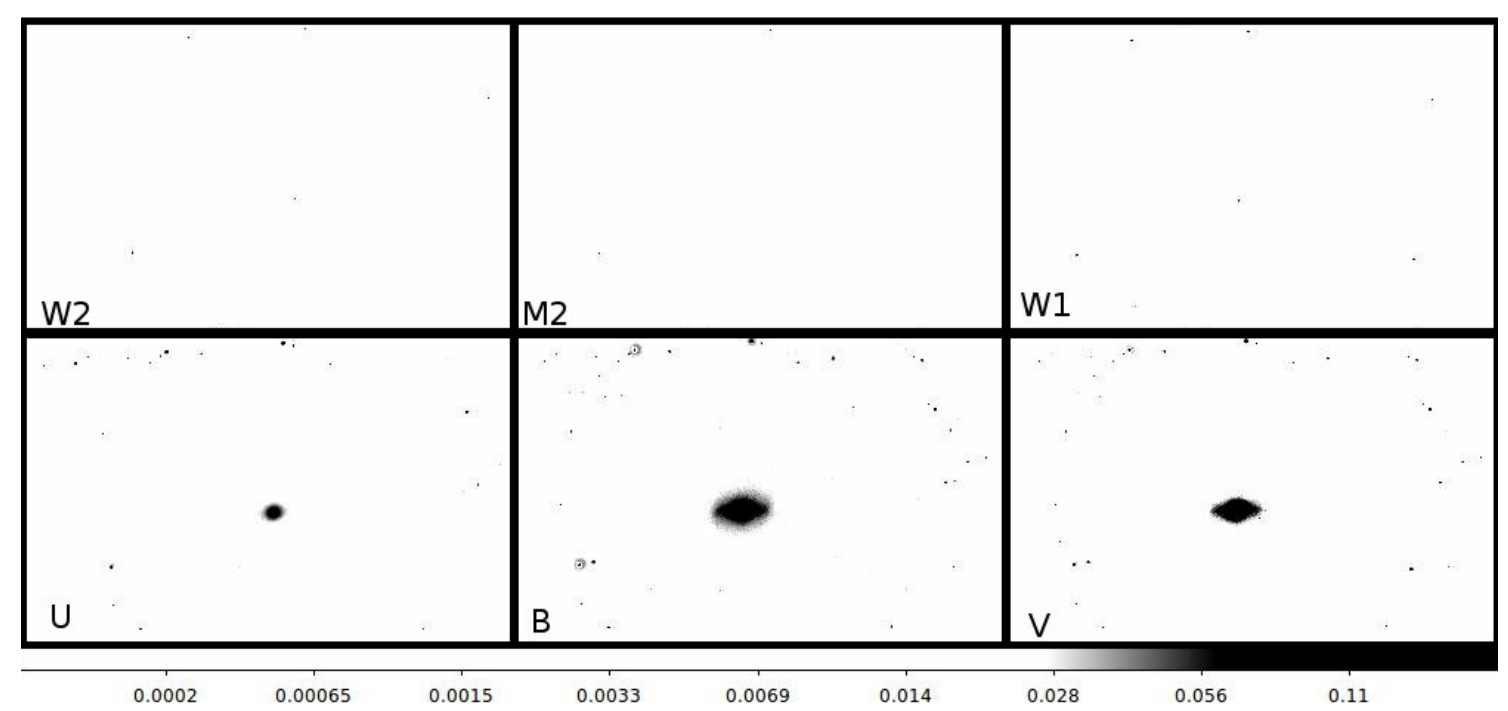

Fig. 1. UVOT count rate images of NGC 1543 by filter (see composite images in Fig. 7 for reference). Following Hoversten et al. (2011), non-white areas have a count rate higher than 0.028 counts s${ }^{-1}$ pixel $^{-1}$, which indicates that coincidence loss effects are larger than $1 \%$ in images binned $2 \times 2$. $U, B, V$ images appear affected by coincidence loss effects, although only at a few percent level. Conversely, NUV images are not affected (see text).

Table 3. NUV and optical integrated magnitudes in the AB system.

\begin{tabular}{lrrrrrr}
\hline \hline Galaxy & $W 2$ & $M 2$ & $W 1$ & $U$ & $B$ \\
\hline NGC 1366 & $17.55 \pm 0.11$ & $17.39 \pm 0.14$ & $16.36 \pm 0.07$ & $14.34 \pm 0.05$ & $13.12 \pm 0.09$ & $12.27 \pm 0.14$ \\
NGC 1415 & $15.89 \pm 0.07$ & $15.88 \pm 0.13$ & $15.00 \pm 0.12$ & $13.77 \pm 0.04$ & $12.43 \pm 0.07$ & $11.65 \pm 0.21$ \\
NGC 1426 & $16.89 \pm 0.23$ & $16.79 \pm 0.11$ & $14.99 \pm 0.15$ & $13.81 \pm 0.06$ & $12.38 \pm 0.07$ & $11.60 \pm 0.09$ \\
NGC 1533 & $15.90 \pm 0.16$ & $16.07 \pm 0.18$ & $14.48 \pm 0.08$ & $13.10 \pm 0.13$ & $11.57 \pm 0.09$ & $10.91 \pm 0.08$ \\
NGC 1543 & $15.36 \pm 0.22$ & $15.64 \pm 0.17$ & $14.28 \pm 0.12$ & $12.87 \pm 0.15$ & $11.25 \pm 0.10$ & $10.60 \pm 0.05$ \\
NGC 2685 & $15.53 \pm 0.07$ & $15.67 \pm 0.13$ & $14.77 \pm 0.13$ & $13.41 \pm 0.17$ & $12.26 \pm 0.13$ & $11.66 \pm 0.07$ \\
NGC 2974 & $16.17 \pm 0.09$ & $16.40 \pm 0.24$ & $15.00 \pm 0.15$ & $13.43 \pm 0.06$ & $11.70 \pm 0.07$ & $10.91 \pm 0.07$ \\
NGC 3818 & $17.17 \pm 0.18$ & $17.34 \pm 0.13$ & $15.73 \pm 0.17$ & $14.10 \pm 0.15$ & $13.14 \pm 0.10$ & $12.11 \pm 0.22$ \\
NGC 3962 & $15.90 \pm 0.15$ & $16.51 \pm 0.11$ & $14.66 \pm 0.11$ & $13.27 \pm 0.09$ & $11.59 \pm 0.12$ & $10.86 \pm 0.08$ \\
NGC 7192 & $16.89 \pm 0.23$ & $16.79 \pm 0.10$ & $15.13 \pm 0.17$ & $13.59 \pm 0.09$ & $12.10 \pm 0.10$ & $11.55 \pm 0.07$ \\
IC 2066 & $16.22 \pm 0.16$ & $16.37 \pm 0.20$ & $15.20 \pm 0.18$ & $13.71 \pm 0.10$ & $12.29 \pm 0.15$ & $11.62 \pm 0.09$ \\
\hline
\end{tabular}

IRAF, increasing the size of the apertures logarithmically. Foreground and background objects have been removed and were substituted with the surrounding background using the IRAF task IMEDIT. Ghosts of bright stars remain a serious problem for an accurate surface photometry. Since the problem only arises in the images ( $W 1$ and $W 2$ bands) of NGC 1366, we have masked them in the same way. To secure a reliable background measure, we performed the measurement well beyond the galaxy emission. ELLIPSE provides the semi-major axis lengths $(a)$, the surface brightness $(\mu)$, the ellipticity $(\epsilon)$, the position angle (PA), and the isophotal shape $\left(a_{4} / a\right)$. This term, labeled $B_{4}$ in the ELLIPSE table, provides the deviation from the elliptical shape, which is parametrized by the fourth cosine coefficient of the Fourier expansion of the residuals of the fitting procedure. The sign, the absolute value, and the behavior of $a_{4} / a$ are indicative of the boxiness $\left(a_{4} / a<0\right)$ or diskiness $\left(a_{4} / a>0\right)$ of the isophotes (Bender et al. 1989; Capaccioli et al. 1990; Governato et al. 1993).

From the surface brightness profiles, we derived apparent magnitudes by integrating the surface brightness within elliptical isophotes. Errors of the NUV and optical magnitudes where estimated by propagating the statistical errors on the isophotal intensity provided by ELLIPSE. Our NUV and optical integrated magnitudes, reported in Table 3, are not corrected for Galactic extinction. NUV luminosity profiles are provided in
Table A.1. This table also lists optical UVOT luminosity profiles in the $V$ band for all galaxies and additionally in the $U$ and $B$ bands for NGC 1366, NGC 2685 and NGC 3818. These profiles are used to complete the structural analysis of our galaxies (see details in Sect. 4.3).

\section{Results}

This section focuses on our main results, which are the comparison of our estimated Swift-UVOT integrated magnitudes with those available in the literature and the analysis of the NUV surface brightness photometry of our ETGs.

\subsection{Integrated magnitudes}

In Fig. 2 we show the comparison of our integrated NUV magnitudes with those obtained by Hodges-Kluck \& Bregman (2014) for NGC 1426, NGC 2974, and NGC 3818. For consistency we corrected our magnitudes for galactic extinction as in Hodges-Kluck \& Bregman (2014), that is, following Roming et al. (2009).

Our W2 magnitudes are fully consistent with Hodges-Kluck \& Bregman (2014) estimates. Our $W 1$ magnitudes are about $1 \sigma$ brighter. $M 2$ values show the largest differences. Our data are almost $2 \sigma$ fainter than in Hodges-Kluck \& Bregman (2014) with the exception of NGC 3818, which is brighter. 

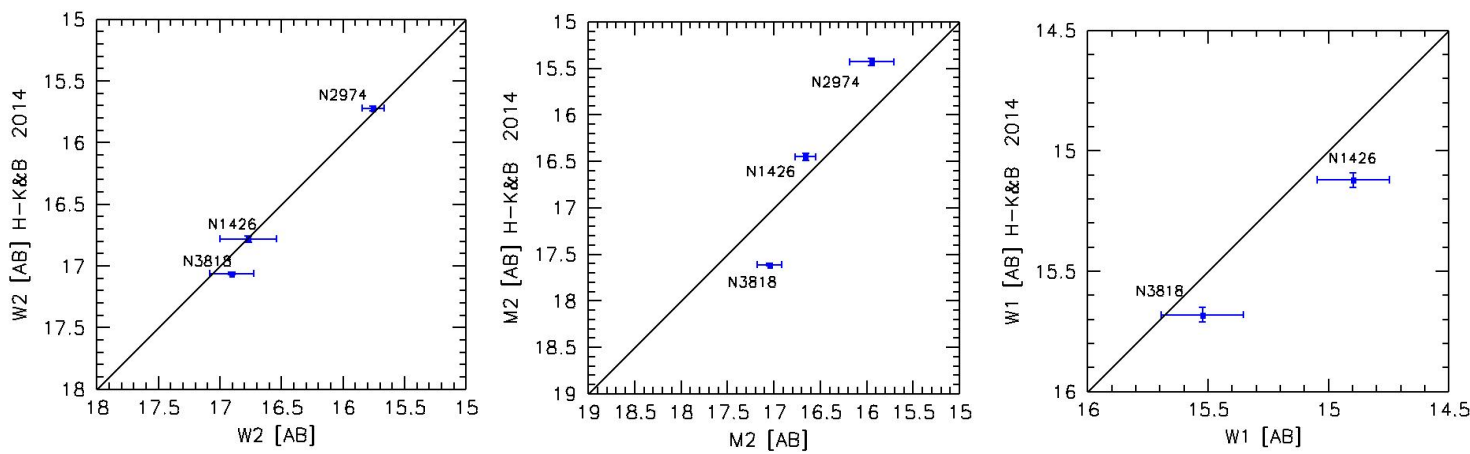

Fig. 2. Our apparent $W 2, M 2$, and $W 1$ integrated magnitudes are compared with those derived by Hodges-Kluck \& Bregman (2014) (galaxies + halo) for the galaxies in common.

However, this difference cannot be due to a ghost in our M2 images (Trinchieri et al. 2015, their Fig. 7) or to the effect of scattered light, which is negligible in the M2 filter (Hodges-Kluck \& Bregman 2014).

We compared our integrated $U, B$, and $V$ magnitudes with the RC3 (de Vaucouleurs et al. 1991) and Hyperleda ${ }^{2}$ catalogs after converting their integrated magnitudes in the AB-system by applying the following correction AB-Vega: $V=-0.01$, $B=-0.13, U=0.79$. As an additional source of comparison, we used the integrated magnitudes provided by the CarnegieIrvine Galaxy Survey (CGS, Ho et al. 2011; Li et al. 2011). This $B, V, R, I$ surface photometric study covers a significant fraction of the present sample and has been performed under good seeing conditions. The comparison is shown in Fig. 3. Most of our integrated magnitudes are consistent within errors with those of the RC3, Hyperleda, and the CGS. There are some discrepant cases. Our $B$ and $V$ integrated magnitudes of NGC 1366 and NGC 3818 are fainter than in the RC3 and the Hyperleda catalogs. We note that the NGC $1543 B$ and $V$ integrated magnitude measures in the CGS are discrepant with both the RC3 and the Hyperleda magnitudes, whose values in turn agree with our measures. We estimate that the coincidence loss in the central part of the $V$ profile amounts to $0.1-0.2$ mag at most (see Table A2 of Karczewski et al. 2013).

We conclude that our NUV and optical integrated magnitudes in general agree with those in the literature. The influence of coincidence loss effect on our optical magnitudes is within estimated photometric errors.

\subsection{NUV-Swift surface photometry}

The top panels of Figs. 4 to 14 compare the optical and the UV morphologies. They show color-composite multiwavelength images of our ETGs. Their luminosity profiles in the $W 2, M 2$, and $W 1$ bands are plotted in the middle panels of these figures and are listed in Table A.1. They are truncated when the uncertainty on the surface brightness exceeds $0.3 \mathrm{mag} \operatorname{arcsec}^{-2}$. As for the integrated magnitudes, they are not corrected for galactic extinction.

To parametrize the shape of our NUV luminosity profiles, we adopted a Sérsic $r^{1 / n}$ law (Sérsic 1968), which is widely used for elliptical and S0 galaxies since it is a generalization of the $r^{1 / 4}$ de Vaucouleurs (1948) law (see, e.g., Caon et al. 1993). Special cases are $n=1$, the value for an exponential profile, and $n=0.5$,

\footnotetext{
2 http://leda.univ-lyon $1 . f r$
}

a Gaussian luminosity profile. Galaxies with $n$ values higher than 1 have a steep luminosity profile in their nuclear regions and extended outskirts. Values lower than 1 indicate a flat nuclear region and more sharply truncated outskirts. From a 2D luminosity profile decomposition of about 200 ellipticals from the SDSS, Gadotti (2009) measured that the Sérsic index in $i$-band has a mean value of $3.8 \pm 0.9$, close to $n=4$, the historical paradigm for bona fide ellipticals (de Vaucouleurs 1948), although with a large scatter.

We best fit a Sérsic law convolved with a point-spread function (PSF) using a custom IDL routine based on the MPFit package (Markwardt et al. 2009) and accounted for errors in the surface photometry. The PSF model is a Gaussian of a given full width at half maximum (FWHM), and the convolution is computed using fast Fourier transformation (FFT) on oversampled vectors. We used the nominal value of the FWHM of the PSF for each UVOT filter. However, as a result of the coadding, binning, and relative rotation of the frames, the FWHM is broadened by $\approx 15 \%$ compared to the nominal value (Breeveld et al. 2010). We verified that the effects of the small variations of the PSF are well within the error associated with the Sersic index. The residuals, $\mu-\mu_{\text {Sersic }}$, are shown in the bottom panels of Figs. 4 to 14 and the Sérsic indices are collected in Table A.2 and are shown in Fig. 15.

The structural properties of our galaxies in the NUV bands are collected in Table A.2. Columns $2-4$ provide the ellipticity, the position angle (PA), and the isophotal shape parameter, $a_{4} / a$, averaged over $a_{80}$ (Col. 6), which is the semi-major axis including $80 \%$ of the total galaxy luminosity. In this average we exclude the values within $2 \times F W H M_{\mathrm{PSF}}$ of the galaxy centers to minimize PSF effects. The quoted error for each measure is the standard deviation around the mean. Figure 16 shows the isophotal shape parameter $a_{4} / a$ as a function of the semi-major axis in the $M 2$ band. The ring- or arm-like structures cause strong variations, therefore we provide the average values of $a_{4} / a$, PA, and ellipticity, as explained above. Table A.2 also reports the Sérsic index resulting from the best fit of the luminosity profile (Col. 5) and the notes about the fit (Col. 7).

We show in Fig. 17 the $(M 2-V)$ color profiles obtained from our UVOT-M2 and $V$ band data, that is to say, we compare data sets with a similar PSFs and avoid using the CGS because of the PSF mismatch. We chose the $M 2$ band over $W 1$ and $W 2$ because it is less strongly affected by scattered light and ghosts (see Sect. 2).

In the next subsection we discuss our NUV photometric results for each single object in the context of the current literature. 

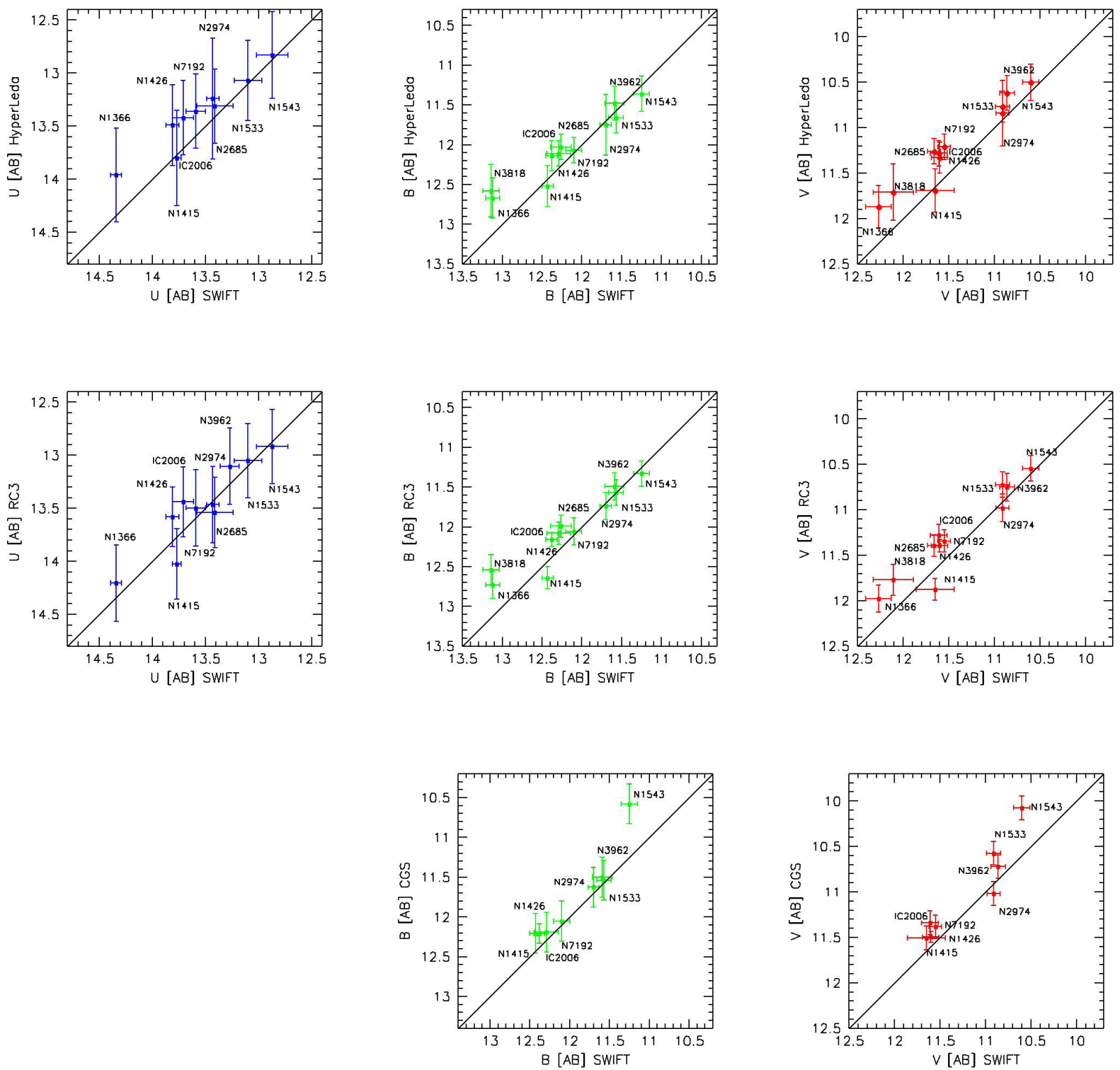

Fig. 3. Comparison between our apparent integrated $U, B$, and $V$ magnitudes and those in the Hyperleda (top panels), the RC3 (de Vaucouleurs et al. 1991) (middle panels), and the CGS (Ho et al. 2011; Li et al. 2011) (bottom panels) catalogs. Magnitudes are given in the $[\mathrm{AB}]$ system. Error bars of the Hyperleda and the RC3 $U[\mathrm{AB}]$ magnitudes account for an additional \pm 0.23 mag to include the uncertainty in the conversion from Vega to $[\mathrm{AB}]$ magnitudes. Magnitudes are not corrected for Galactic extinction.

\subsection{Sérsic index analysis}

The study of the Sérsic index is usually performed on unstructured ETGs such as NGC 1366, NGC 1426, NGC 3818, NGC 3962, and NGC 7192. ETGs with ring- or arm-like structures have either knotty, irregular, or filamentary morphologies, as expected for sites hosting young stellar populations. These features reflect on their luminosity profiles, and sometimes they become the dominant characteristics in the NUV luminosity profile. We are interested in obtaining a description of the galaxy structure underlying these features, however, which may differ when moving from UV to optical bands. For this reason, we fit and compare Sérsic indices in different bands and consider case by case the regions where these features occur (see Sect. 4.4). This strategy allows us to follow the variation of the underlying galaxy structure, if any, across different wavelengths.

For several reasons we decided to use CGS data (Ho et al. 2011; Li et al. 2011) to investigate the variation in Sérsic index with wavelength. All galaxies except for NGC 1366, NGC 2685, and NGC 3818 have $B, V, R, I$ photometry in the CGS that was taken with good seeing conditions. They have a median FWHM of the seeing of $1^{\prime \prime} 17,1^{\prime \prime} 1,1^{\prime \prime} 01$, and $0^{\prime \prime} \cdot 96$ in the $B, V, R$, and $I$ bands, respectively, which is better than our UVOT $B(F W H M=$ 2 ".18) and $V(F W H M=2$ '!19). In a few cases the CGS optical luminosity profiles extend farther than the profiles we obtained. Finally, the $R$ and $I$ filters allow us to investigate the galaxy photometric structure from NUV to NIR. For NGC 1366, NGC 2685, and NGC 3818, which are not part of the CGS sample, we used our UVOT $U, B, V$ luminosity profiles to complete the analysis of the sample (see Table A.1).

To be consistent with our analysis of the UV data, we did not follow the multicomponent decomposition approaches discussed in the literature such as fitting the profile with multiple Sersic law profiles by Huang et al. (2013) (see also Laurikainen et al. $2006,2010,2011)$. We performed the analysis of the CGS profiles with a single Sérsic law fit, convolving the profile with the median FWHM of the seeing. This choice allows us to use the same criteria as adopted for the NUV, that is, masking the same 
R. Rampazzo et al.: Investigating ETGs evolution with a multiwavelength approach. II.
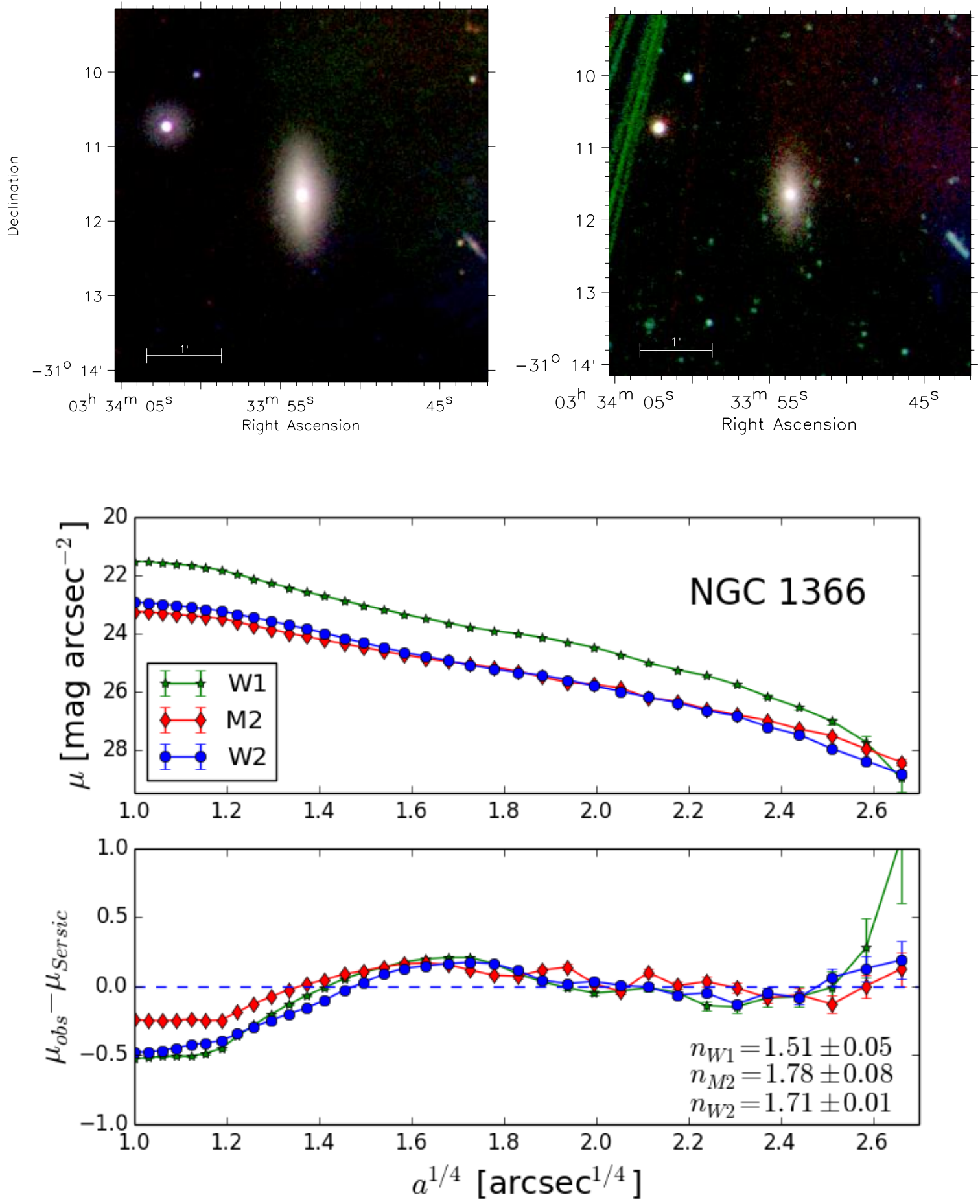

Fig. 4. NGC 1366. Top panels: color-composite image in the $U, B, V$ filters (left: $U=$ blue, $B=$ green, $V=$ red) and $W 1, M 2$ and $W 2$ filters (right: $W 2$ = blue, $M 2$ = green, $W 1=$ red). The field of view is $5^{\prime} \times 5^{\prime}$, north is at the top, east to the left. Middle and bottom panels: $W 1$, M2, and $W 2$ luminosity profiles and residuals from their best fit with a single Sérsic law accounting for the filter PSF.

structures, and extending the fit to the same regions (see notes in Col. 8 of Table A.2) for 9 out of 11 galaxies. To map the underlying galaxy structure for the remaining 2 galaxies, namely NGC 1533 and NGC 2974, we masked the substructures that are present in optical but not in the NUV profiles, or vice versa, as explained in the individual notes of these galaxies.

The synoptic view of the results of the single Sérsic fit for the different bands, from NUV to NIR, is shown in Fig. 15. This figure shows that Sérsic indices vary from filter to filter for a given galaxy, and they also vary from galaxy to galaxy. Only few of the indexes plotted comply with the canonical value of $n=4$, which is highlighted with the arrow in the figure. Before entering in the discussion of these results in the context of the current literature, we discuss the NUV photometric results in detail for each galaxy in the next subsection.

\subsection{Notes on individual galaxies}

NGC 1366. A bright star is present to the north. Light scattered by the instrument and a ghost of the star projects in the northwest 
region around the galaxy in the $W 1$ the $W 2$ bands. The irregular background could perturb our photometric study in these two bands.

Both the RC3 (de Vaucouleurs et al. 1991) and the RSA (Sandage \& Tammann 1987) classify NGC 1366 as an S0. Recently, Buta et al. (2015) classified NGC 1366 as SA(rl)0 $0^{-}$sp in the survey $\mathrm{S}^{4} \mathrm{G}$, adding the note that a very subtle inner ringlens (rl) is present (see also Comerón et al. 2014). Our surface photometry extends out to $50^{\prime \prime}(\sim 5 \mathrm{kpc}$ using the distance in Table 1), which is about $2 \times r_{\text {eff }}$ in NUV.

The $n$ values (Fig. 4 bottom panel) are lower than 2 in all the NUV bands, consistent with the presence of a disk structure. Very similar values are also measured in the $U$ and $V$ bands, while the $B$ band gives a higher value of $n=2.55 \pm 0.03$ (Fig. 15). The nuclear part of the galaxy is brighter than the Sérsic model in all the NUV bands.

The $a_{4} / a$ ratio in $M 2$ (Fig. 16) shows a boxy nucleus and disky outskirts.

The $(M 2-V)$ color profile of this galaxy (Fig. 17) is bluer in the outer than in the inner parts.

NGC 1415. Our photometry extends out to $\sim 16.5 \mathrm{kpc}$ in radius, $4 \times r_{\text {eff }}$ on average in our NUV bands. The optical and NUV images (Fig. 5, top panels) show the complex structures present in the galaxy, which justify its controversial classification: an S0 for the RC3 and a spiral for the RSA. A complex system of dust is also visible in the optical composite image of the CGS (Ho et al. 2011). More recently, Laurikainen et al. (2011) classified the galaxy as (RL)SABa (r'l, nr)0+ in their Near Infrared Atlas. Buta et al. (2015) assigned a morphological type $T=-1$, adding the notation "grand-design spiral".

In short, recent classifications tend to describe the galaxy as an S0 with an outer ring-lens (RL) system, without bar, with an inner ring-lens (r'l) and a nuclear ring (nr). The system of rings dominates our NUV images, especially in the $M 2$ and $W 2$ filters, and reflects on the shape of the luminosity profiles: there is no luminosity peak in the center since the luminosity of the nuclear ring prevails. The outer ring, well visible in the NUV bands, shows a position angle different from the inner one, as pointed out by Comerón et al. (2014). We measured the position angle, PA, and, at the outer edge of the NUV features, the semi-major, $a$ and semi-minor, $b$, projected axes. The nuclear ring (nr) has PA = $166^{\circ} \pm 2^{\circ}, a=10.8^{\prime \prime}, b=5.2^{\prime \prime} ;$ the r'l PA $=139^{\circ} \pm 2^{\circ}, a=50^{\prime \prime}$, $b=14^{\prime \prime}$; the RL PA $=150^{\circ} \pm 2^{\circ}, a=152.8^{\prime \prime}, b=60.3^{\prime \prime}$.

The ring- or arm-like features perturb the entire NUV luminosity profiles. Without masking any feature, the Sérsic indexes show a large variation $0.85<n<2.46$. The range of $n$ values highlights the presence of an underlying disk structure.

The $B, V, R, I$ bands in the CGS cover an area radially more extended than our NUV profiles. The indices summarized in Fig. 15 are the result of a fit considering the CGS profile out to the NUV outermost observed radius. Moreover, the fit of the entire CGS profiles provides similar results that are comparable within the errors. The Sérsic index ranges from $2.83 \pm 0.04$ to $3.25 \pm 0.38$. Values of the Sérsic index $n \geq 3$ argue against a disk morphology, although the galaxy shows a ring that reveals the presence of the disk.

The color profile $(M 2-V)$ in Fig. 17 shows strong variations that are due to the ring- or arm-like structures. The color is bluer in the inner regions, notwithstanding the dust that is clearly visible in our images, and it is redder in the outskirts.
NGC 1426. The galaxy is considered a bona fide elliptical by both the RC3 and the RSA catalogs. The E classification was questioned by Capaccioli et al. (1988), who studied its geometrical structure down to $\mu_{B} \approx 28 \mathrm{mag} \operatorname{arcsec}^{-2}$. The authors suggested that NGC 1426 is an S0 since the galaxy does not show any significant isophotal twisting $\langle\mathrm{PA}\rangle=105^{\circ} \pm 1^{\circ}$ out to $100^{\prime \prime}$ $(\mathrm{PA}=103.5 \pm 1.2$ in Ho et al. 2011), as expected for disk galaxies. Huang et al. (2013) modeled this galaxy with three Sérsic components with $n=2.17,0.69,2.13$. There is no comment about a possible galaxy reclassification in their Table 1 on the basis of these low $n$ values. Hopkins et al. (2009) provided a Sérsic fit of the $V$-band optical profile that combines high-resolution inner galaxy regions from HST and ground-based measurements from different sources. They propose a two-component fit, "Sérsic + cusp", with a value of the Sérsic index $5.26_{-0.35}^{+0.11}$ (see their Table 1).

Our integrated NUV magnitudes (Table A.2) agree well with those in Hodges-Kluck \& Bregman (2014). The bottom panel of Fig. 6 shows that our NUV luminosity profiles do not present peculiar features. The range of indices obtained with a single Sérsic fit out to $a \sim 90^{\prime \prime}$ is $2.65 \leq n \leq 2.86$.

The CGS surface photometry extends out to $\sim 230^{\prime \prime}$, although with large photometric errors in the outskirts, which are well beyond our NUV data. The value of our Sérsic fit of the CGS data over the same range as our NUV analysis outlines values lower than the classic $r^{1 / 4}$ law, just as in the NUV bands (Fig. 15).

The $(M 2-V)$ color profile of this galaxy (Fig. 17) is rather flat. The $a_{4} / a$ isophotal shape profile in the $M 2$ band is very noisy in the outskirts and shows some boxy isophotes in its intermediate part. In the optical Li et al. (2011) showed $a_{4} / a$ (their $B_{4}$ ) consistent with 0 out to $100^{\prime \prime}$.

NGC 1533. In the RSA catalog this galaxy has an uncertain classification, barred S0 or spiral, while it is a barred S0 in the RC3 catalog. The classification of Laurikainen et al. (2011), (RL) $\mathrm{SB} 0^{\circ}$, confirms the RC3 classification and indicates the presence of an RL associated with the barred S0 (Fig. 7). Rings are also discussed in Comerón et al. (2014). A Sérsic index value $n=1.5$ has been obtained by Laurikainen et al. (2006) with a 2D fit of the bulge, which they considered as a pseudo-bulge. GALEX images show a far-UV bright incomplete ring- or armlike feature at a radius of $\sim 45^{\prime \prime}-55^{\prime \prime}$ (see, e.g., Marino et al. $2011 \mathrm{c}$, and references therein) with a bright knotty structure. This feature is also visible in our Swift-UVOT images. In particular, the top right panel of Fig. 7 shows that the UV bright structures are reminiscent of arms. Approximating the edge of the UV bright northwest "arc" with an ellipse having PA $=120^{\circ} \pm 2$ and semi-major and semi-minor axes $a=58^{\prime \prime}$ and $b=45^{\prime \prime}$, we note that this ellipse does not include the UV bright arm-like structure in the southwest. These arm-like structures are predominant features in the NUV luminosity profiles (Fig. 7, middle panel).

The single Sérsic fit shown in Fig. 7 (bottom panel) includes the NUV bright structures. We adopt these values of the fit and report them in Table A.2 and Fig. 15. The residuals from the fit emphasize the regions that are covered by the ring- or arm-like features, which are seen as excesses of light. The nucleus itself shows an excess of light with respect to the fit.

The bar is not as evident in the NUV (see also the $a_{4} / a$ profile in Fig. 16) as it is in the optical bands. In order to map the underlying galaxy structure, we decided to mask the CGS $B, V, R$, $I$ luminosity profiles in the region from $20^{\prime \prime}$ to $90^{\prime \prime}$, corresponding to the bar and the lens, and obtained the $n$ values shown in Fig. 15. We tested the reliability of the range of the Sérsic index 
R. Rampazzo et al.: Investigating ETGs evolution with a multiwavelength approach. II.
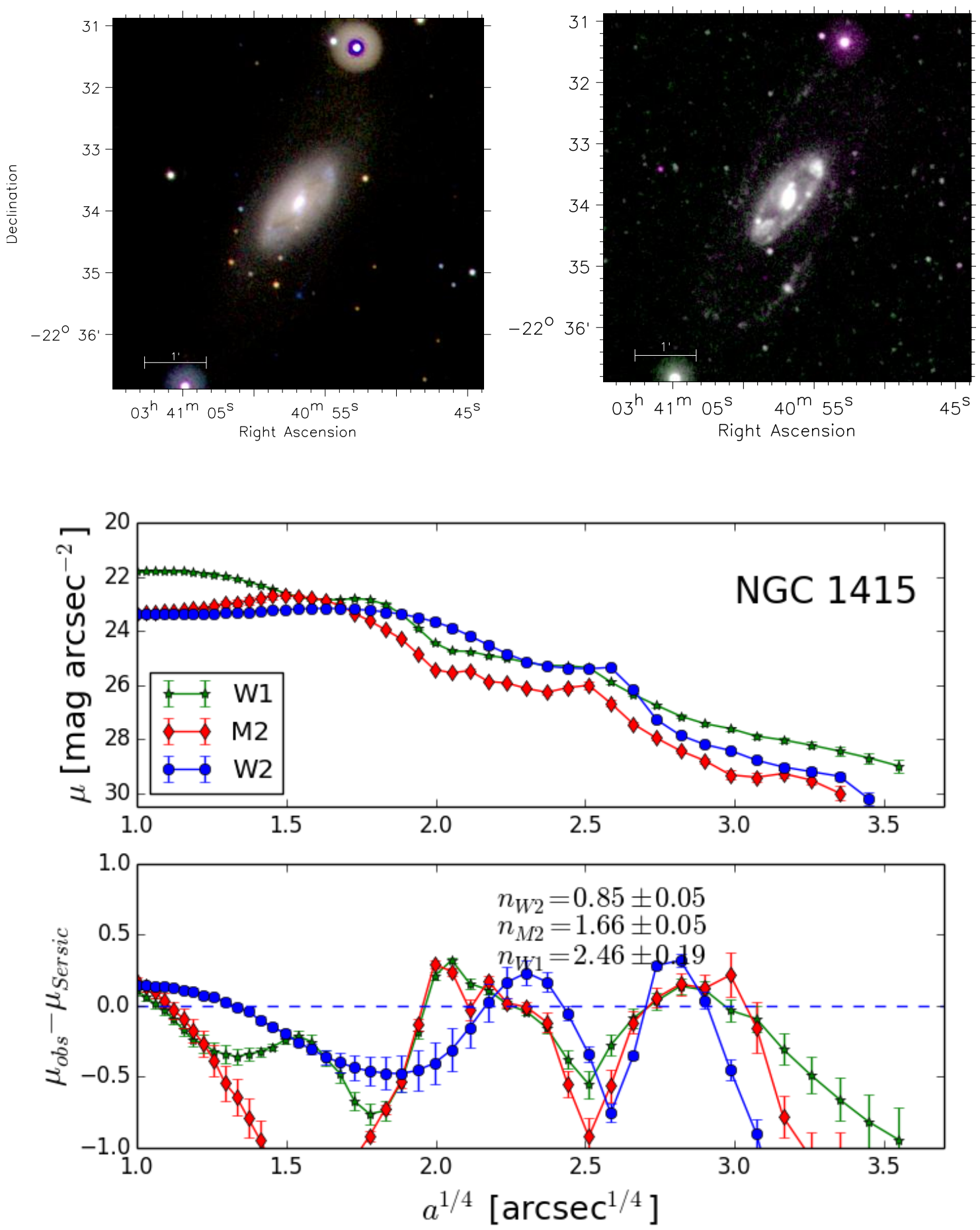

Fig. 5. As in Fig. 4 for NGC 1415. The field of view is $7^{\prime} \times 7^{\prime}$. The wide rings around bright sources in the left panel are an artifact of the UVOT on-board centroid algorithm in the high-count-rate regime (see also Hoversten et al. 2011, and references therein).

variation by repeating the fit of the NUV bands and masked the region from $20^{\prime \prime}$ to $90^{\prime \prime}$, similar as in the optical wavelengths. The Sérsic indices vary from $2.14 \pm 0.06$ to $n=3.69 \pm 0.07$ in $W 2$ and $W 1$, respectively, which means that the values are similar to those in the unmasked NUV fits reported in Fig. 7. We therefore used the unmasked Sérsic fit values for our analysis. To summarize our findings on the values of the Sérsic index, we select the range from $n \sim 2.54$ (M2 band) to 4.89 ( $V$ band) as the best representation of the underlying galaxy structure.

The $(M 2-V)$ color profile (Fig. 17) becomes bluer with increasing galactocentric distance beyond $16^{\prime \prime}$. Knots and the arm-like structures discussed above appear bluer than the galaxy body.

NGC 1543. The galaxy is seen nearly face-on and is classified as a barred S0 in both the RSA and the RC3. Laurikainen et al. (2011) classified the galaxy as $(\mathrm{R}) \mathrm{SB}(1, \mathrm{nl}, \mathrm{nb}) 0^{+}$, which indicates the presence of a inner lens (l), a nuclear lens (nl), and a nuclear bar (nb) in addition to the bar reported by the RC3 and the RSA, and the outer ring (R). Erwin et al. (2015) decomposed the galaxy luminosity profile with a Sérsic law+exponential and reported a disk and a composite bulge with $n=1.5$. 

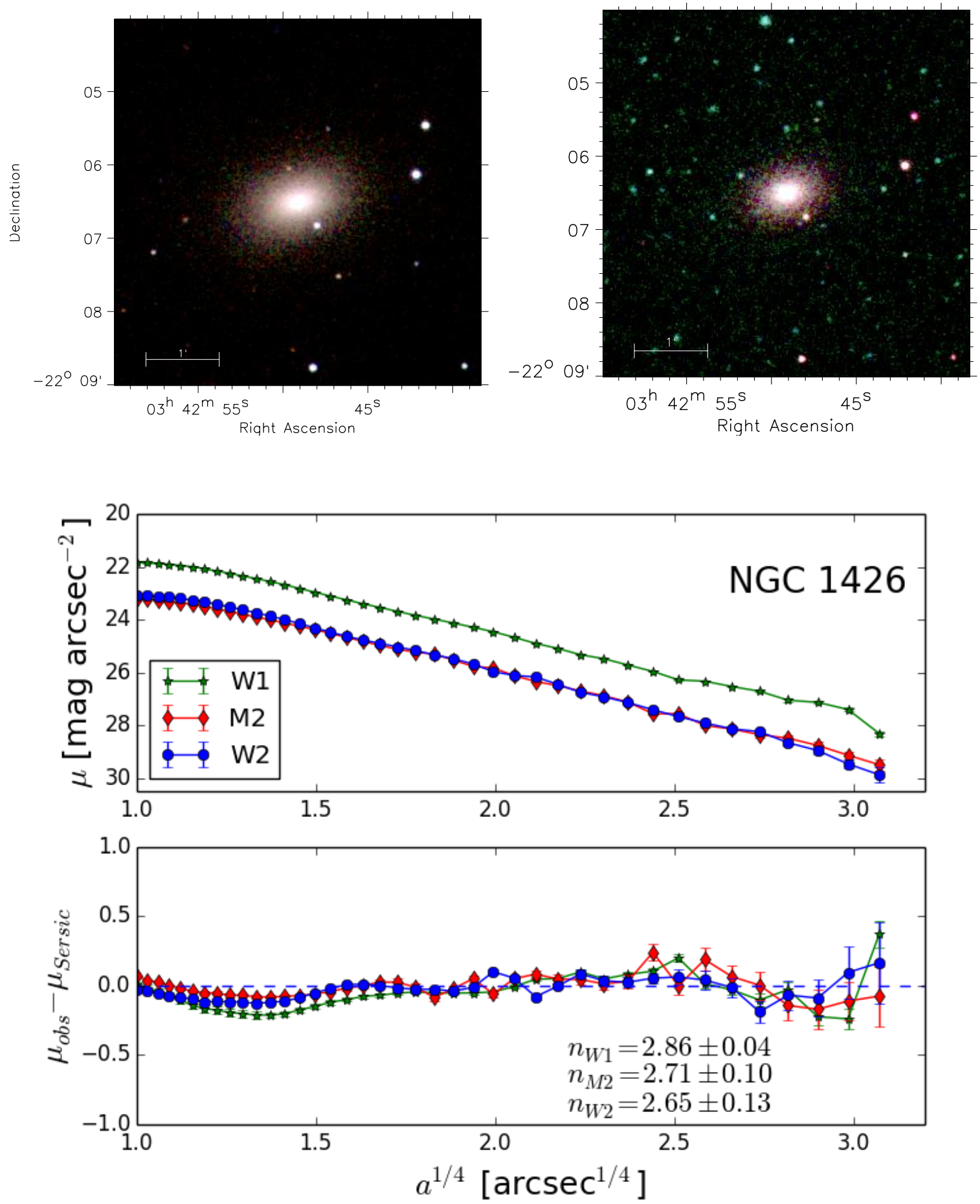

Fig. 6. As in Fig. 4 for NGC 1426. The field of view is $5^{\prime} \times 5^{\prime}$.

The inner and outer bars as well as the lenses are visible in the UVOT optical and NUV images. The outer ring, faint and diffuse in the optical images, becomes brighter in the NUV. Starting from $U$ to $W 2$, a filamentary arm-like or ring structure emerges at the outer edge of this ring (Fig. 8 top right panel).

The NUV luminosity profiles and the values of Sérsic fit are shown in the bottom panels of Fig. 8. We exclude from the single Sérsic fit the ring- or arm-like structure in the galaxy outskirts. The fit is still poor because the structure of the galaxy out to its outskirts is complex (see also the $a_{4} / a$ profile in Fig. 16). The NUV Sérsic indices are lower than 3 , and the galaxy nucleus is brighter than the Sérsic model in the NUV filters. In the $B, V, R$, and $I$ bands the Sérsic fit shows that the luminosity profiles are consistent with an $r^{1 / 4}$ law (Fig. 15).

The $(M 2-V)$ color profile shown in Fig. 17 reddens out to 57", where it starts to become bluer. Colors as blue as $M 2-V \simeq 4$ are found for the arm-like structure at $r>80^{\prime \prime}$.

NGC 2685. The galaxy is known as the Helix galaxy. RSA classifies this peculiar object, which shows polar rings and polar dust-lanes, as $\mathrm{SO}_{3}$ pec. Its polar rings, arcs, and blobs, well known in the optical bands, become quite spectacular in NUV filters (Fig. 9, top right panel). 
R. Rampazzo et al.: Investigating ETGs evolution with a multiwavelength approach. II.
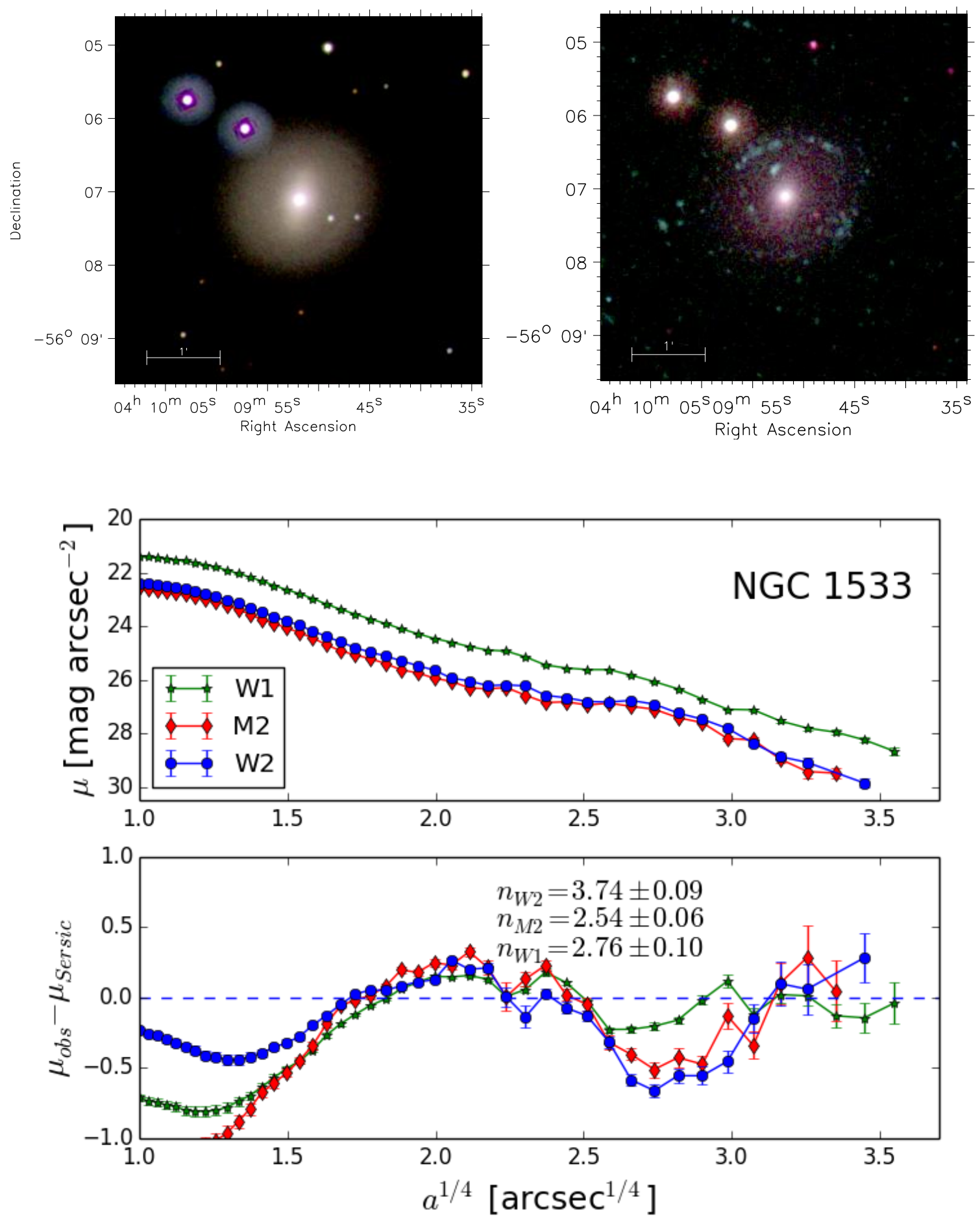

Fig. 7. As in Fig. 4 for NGC 1533.The field of view is $5^{\prime} \times 5^{\prime}$.

The NUV emission is very irregular in all UVOT filters. As a consequence, we performed a Sérsic fit to the NUV luminosity profiles without masking any component (see bottom panel of Fig. 9). Since optical luminosity profiles are not available in the literature, we used UVOT $U, B$, and $V$ images to derive luminosity profiles and to estimate the Sérsic indexes, without masking any component as in the NUV. Figure 15 shows the results: all the UVOT bands have very similar values, $n \lesssim 2.5$, suggesting an extended disk at all wavelengths. A deep optical view of this galaxy is presented by Duc et al. (2015) that confirms the picture of an extended low surface brightness disk and ring- or arm-like structures (see the top left panel in their Fig. 17). Therefore it is not surprising that the $(M 2-V)$ color of this galaxy becomes bluer, from $\sim 6$ up to $\sim 2$, with increasing galactocentric distance (Fig. 17).

NGC 2974. This galaxy is classified as E4 in both the RSA and the RC3. However, Buta et al. (2015) classified it as SA(r)0/a with type $T=0.0$. A bright star superposed on the SW side of the galaxy hampers the study of this galaxy mainly at optical wavelengths.

The CGS optical image atlas shows a faint extended ring- or arm-like structure in NGC 2974 (Ho et al. 2011; Li et al. 2011). 

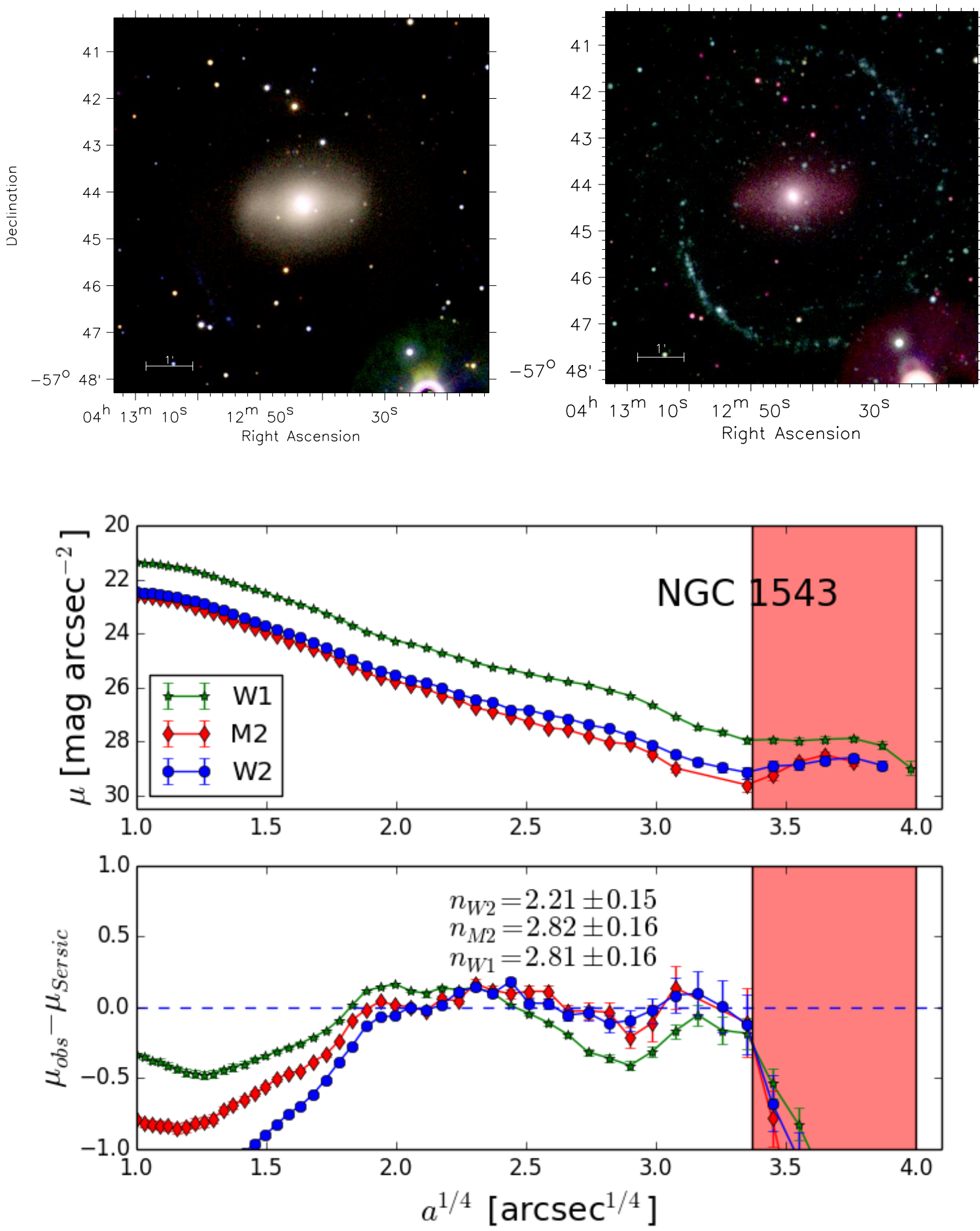

Fig. 8. As in Fig. 4 for NGC 1543. The field of view is $8^{\prime} \times 8^{\prime}$. The shaded area indicates the masked region in the Sérsic law fit (see text).

This structure is very bright in the GALEX far-UV (Jeong 2009; Marino et al. 2011a,b,c) and in our NUV images (top right panel of Fig. 10).

The CGS optical and NIR luminosity profiles do not show signatures of this structure, which appears in our NUV luminosity profiles. To describe the galaxy underlying structure from NIR to NUV, we considered two strategies.

We obtained the NUV Sérsic indices both by masking the luminosity profile from $30^{\prime \prime}$ to $100^{\prime \prime}$ where the ring- or arm-like features are prominent and without masking, as in the optical.
In the first case, the NUV Sérsic indices we derived are very similar to each other, ranging from $3.23 \pm 0.15$ in the $M 2$ band to $3.43 \pm 0.10$ in the $W 1$ band (these are reported in Fig. 10, bottom panel, and in Fig. 15). In the second case, that is, without any masking, we derive $n \lesssim 2.5$, suggesting that the NUV emission marks a disk structure.

The Sérsic indices in NIR and optical bands from the CGS profiles range from 2.9 to 4.2 (see Fig. 15). Hopkins et al. (2009) fit a cusp+Sérsic laws and reported a value of the Sérsic index of $4.06_{-0.48}^{+0.77}$ in the $V$ band. 
R. Rampazzo et al.: Investigating ETGs evolution with a multiwavelength approach. II.
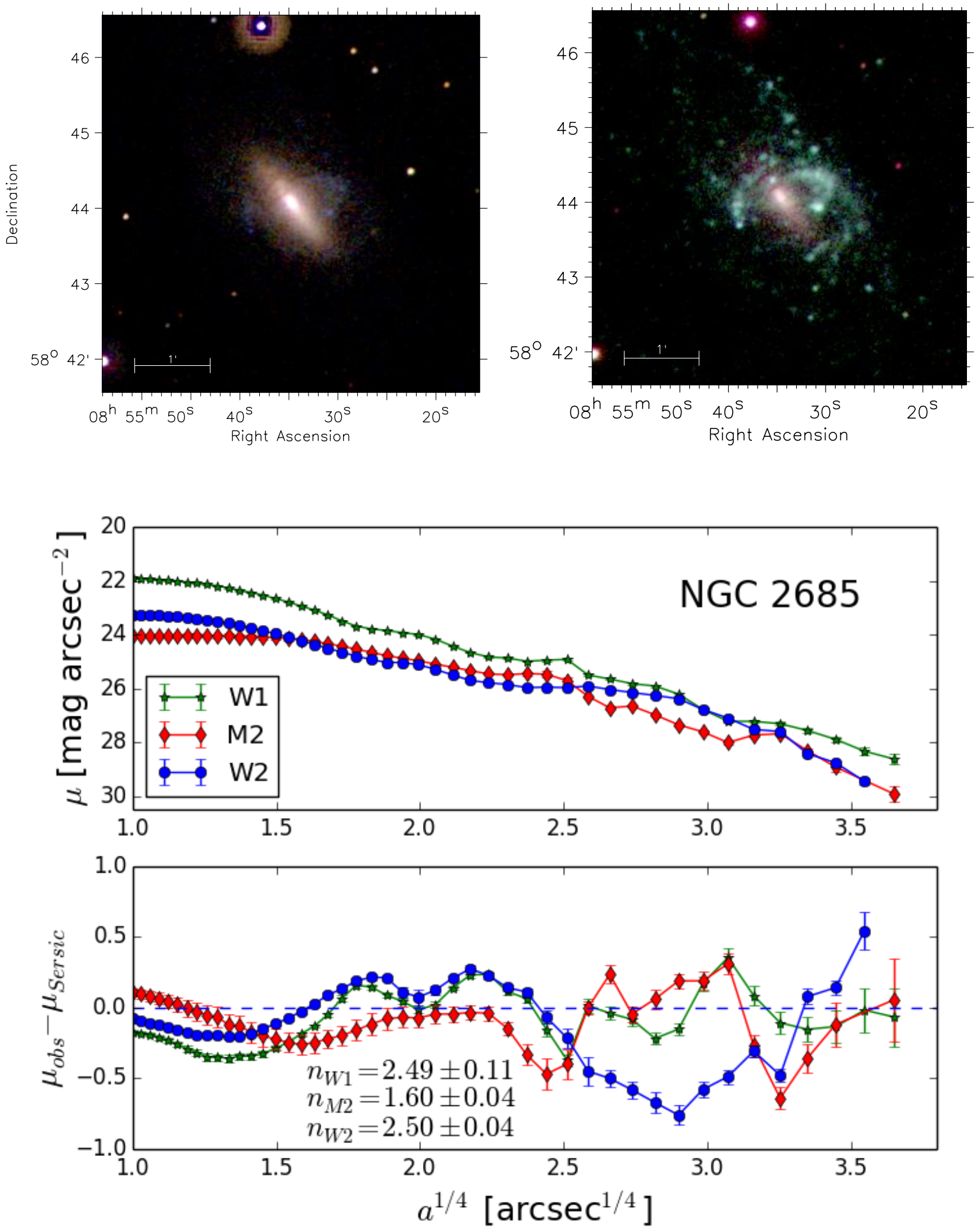

Fig. 9. As in Fig. 4 for NGC 2685. The field of view is $5^{\prime} \times 5^{\prime}$.

The blue arm-like structure starts to emerge at $\approx 30^{\prime \prime}$ in the $(M 2-V)$ color profile of Fig. 17.

NGC 3818. This galaxy is classified E5 in the RSA and the RC3. A more detailed description is given by Scorza et al. (1998), who considered NGC 3818 to be a bulge-dominated ETG with a disk that is fully embedded in a boxy bulge.

Our optical images do not show remarkable features (top left panel of Figs. 11). The Sérsic index from NUV luminosity profiles is in the range $2.73 \pm 0.18<n<3.59 \pm 0.25$. Since the galaxy does not belong to the CGS sample, we used our UVOT data set to study the optical wavelengths.

Our Sérsic index estimate in the UVOT- $V$ band is $3.09 \pm 0.17$ (Fig. 15) in agreement with the value of Hopkins et al. (2009), $n=2.81_{-0.06}^{+0.06}$, in the same band.

We found that the galaxy is slightly disky, as shown in Fig. 16 and Table A.2. Figure 17 shows that this galaxy has a red color profile, almost constant around an average value, $(M 2-V) \sim 5.1$. 

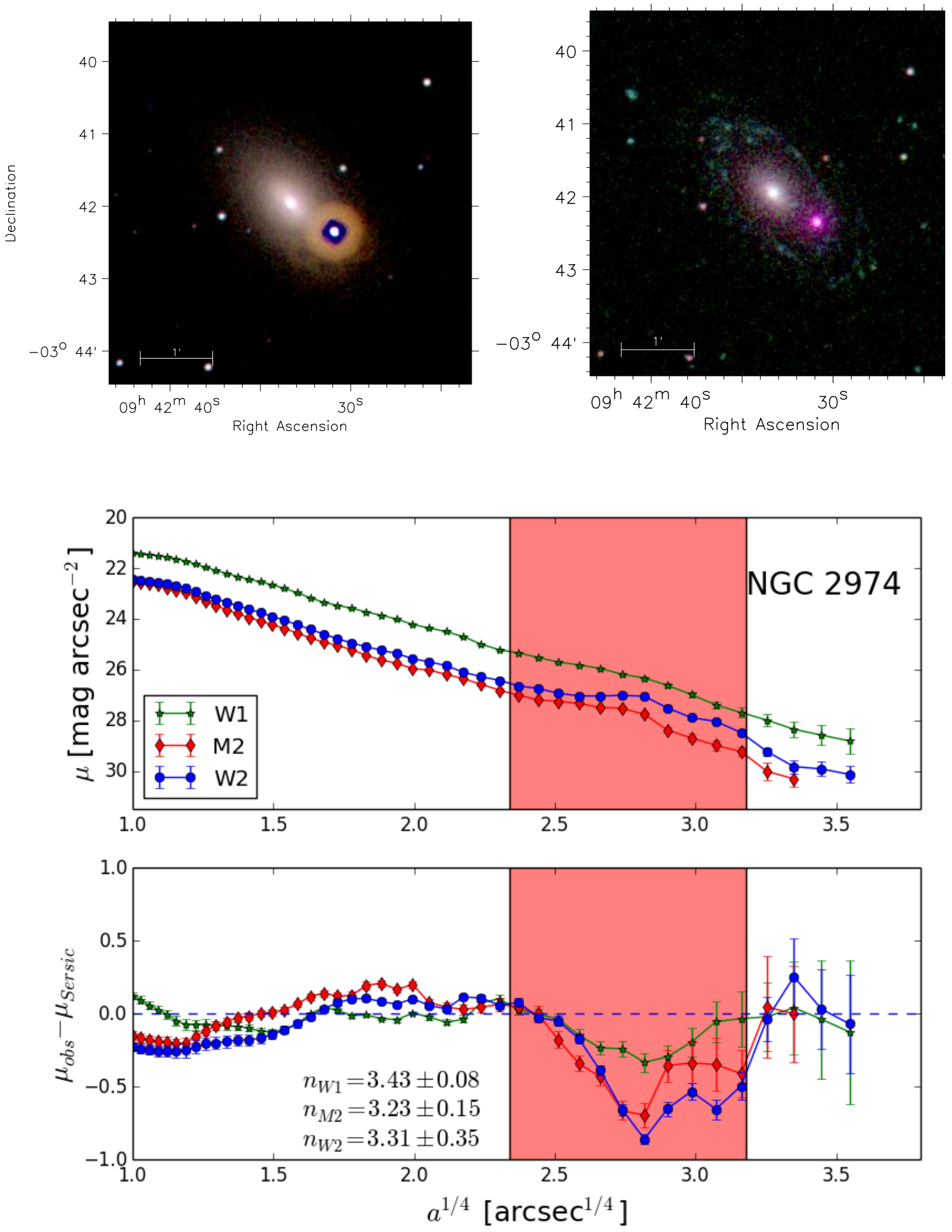

Fig. 10. As in Fig. 4 for NGC 2974. The field of view is $5^{\prime} \times 5^{\prime}$. The shaded area indicates the masked region in the Sérsic law fit (see text).

NGC 3962. The galaxy is classified E1 in the RSA and the RC3 and E+3-4 by Buta et al. (2015). In the NUV bands we find that its average ellipticity is in the range $\epsilon_{80} \sim 0.18-0.24$ (Table A.2).

The galaxy does not show an obvious disk structure, although the value of the $a_{4} / a$ isophotal shape profile tends to increase toward the outskirts. Furthermore, the NUV luminosity profiles show two distinct trends with a break at $a^{1 / 4}=2.45 \operatorname{arcsec}^{1 / 4}$. This break, which is also visible at the same radius in the CGS luminosity profiles, is emphasized by our Sérsic fit. Sérsic index values range from 2.52 to 3.28 in the NUV and from 3.10 to 4.41 in the optical bands (see Fig. 15). Huang et al. (2013) found that the $V$-band profile is well fitted with three Sérsic laws with indices 3.25, 0.51, and 1.46. The best fit of the sum of a cusp plus Sérsic law in Hopkins et al. (2009) 
R. Rampazzo et al.: Investigating ETGs evolution with a multiwavelength approach. II.
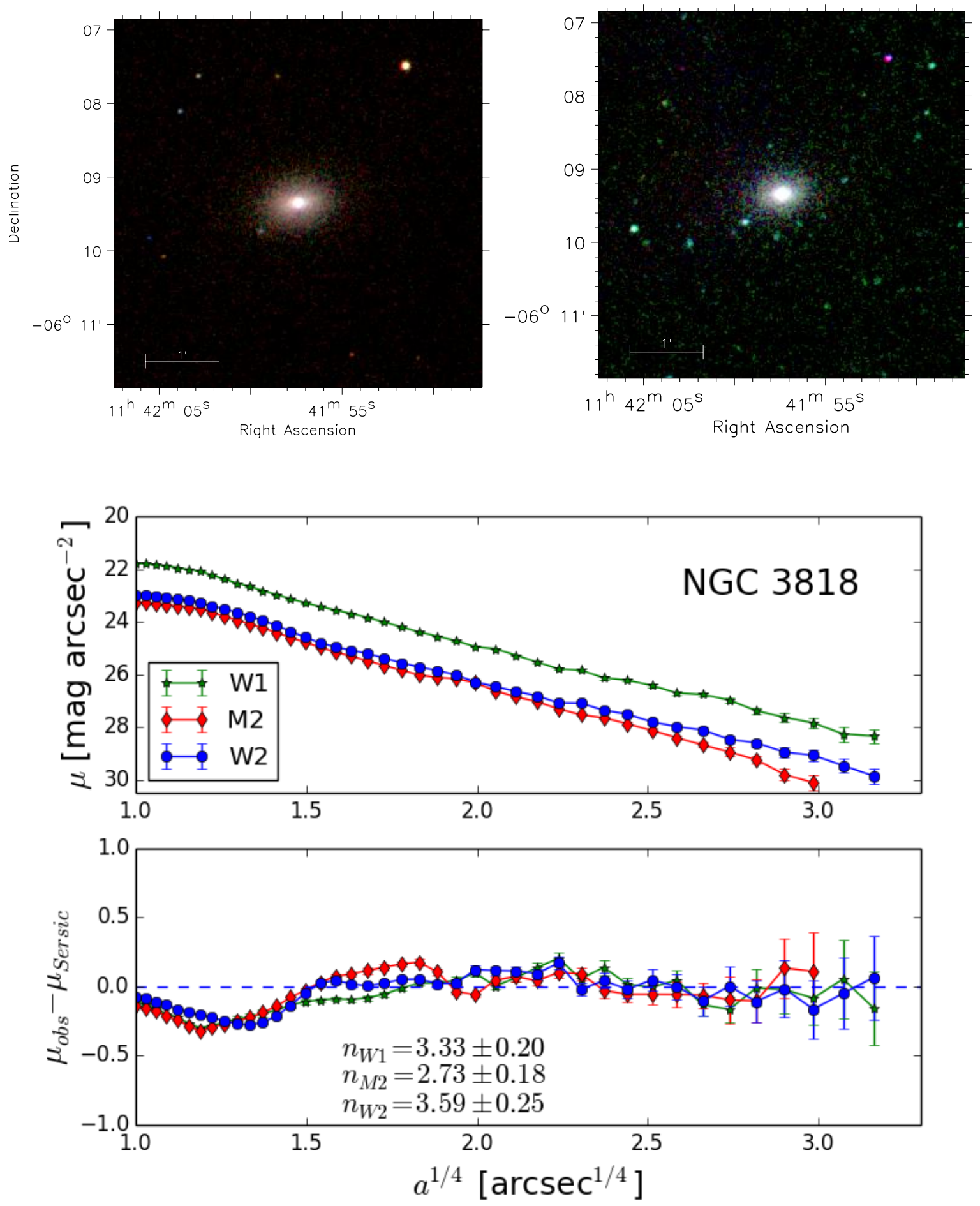

Fig. 11. As in Fig. 4 for NGC 3818. The field of view is $5^{\prime} \times 5^{\prime}$.

in the same band gives $n=3.74$. The 2D single Sérsic fit of Salo et al. (2015) is unsatisfactory $(n=6.1)$, leaving space for a better fit that includes additional components.

Figure 17 shows that this galaxy has quite a red profile that is constant around $(M 2-V) \sim 5.3$ out to the galaxy outskirts.

NGC 7192. The galaxy is classified S0 in the RSA and elliptical (.E+..*.) in the RC3. Huang et al. (2013) suggested that the galaxy is an S0 on the basis of a multiple Sérsic fit. Their four-component model recognizes a bulge $(n=1.7)$, two lenses
( $n=0.4$ and $n=0.5)$, and a disk $(n=0.9)$. The presence of two lenses in addition to an outer exponential disk has previously been revealed by Laurikainen et al. (2011). The Huang et al. (2013) model and that of Laurikainen et al. (2011) converge in indicating that this galaxy is an S0 rather than an E (Huang et al. 2013).

Our fits of the NUV luminosity profiles (Fig. 13 bottom panel) provide values of $n$ that are slightly shallower $(n \approx 3)$ than the $r^{1 / 4}$ law. Similar values are derived in the optical and NIR bands (Fig. 15). The nucleus is underluminous with respect to the Sérsic fit both in the $W 2$ and $M 2$ bands. 

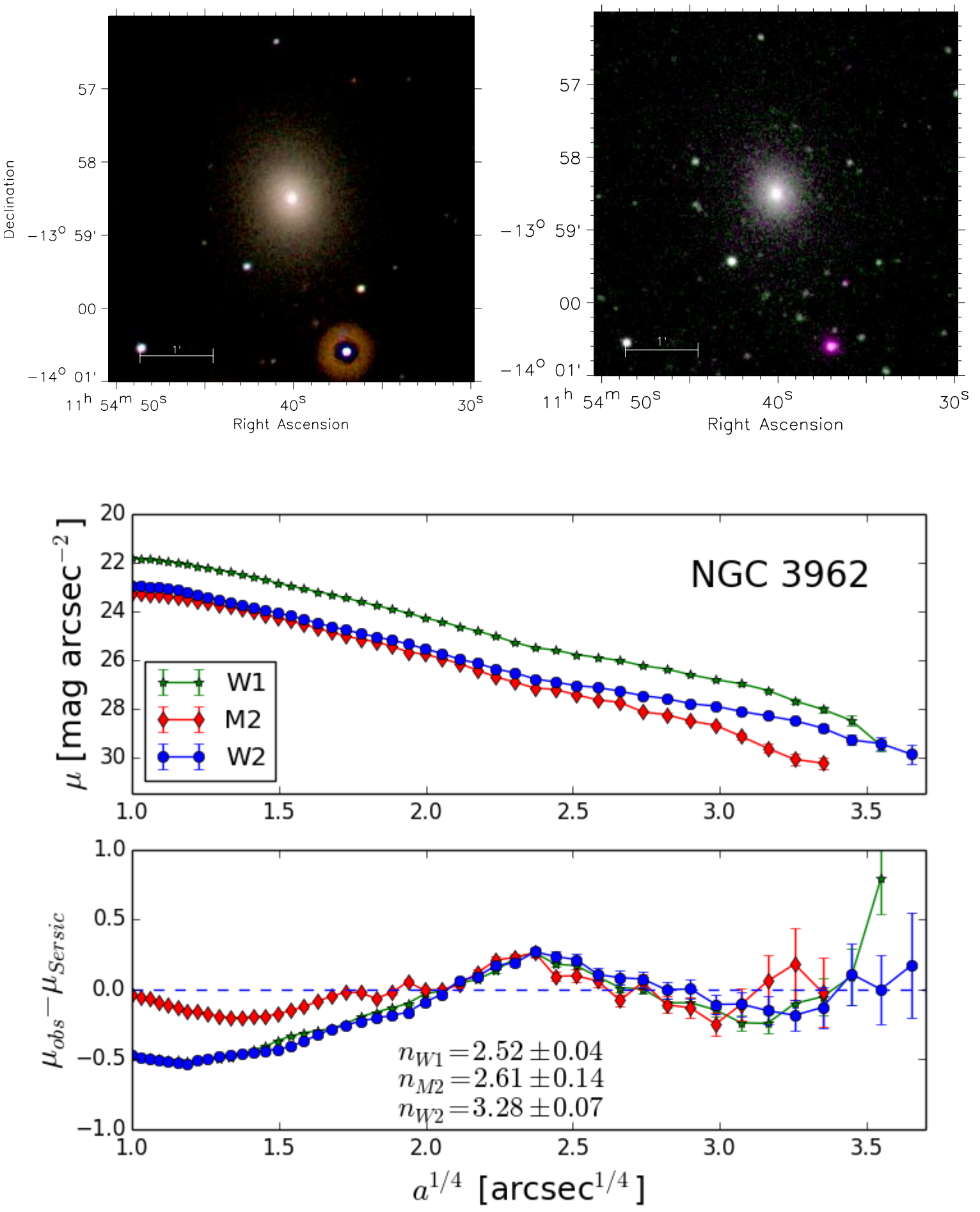

Fig. 12. NGC 3962. As in Fig. 4. The field of view is $5^{\prime} \times 5^{\prime}$.

We point out that NUV images highlight a blue knot at 17 ".5 northwest of the galaxy center that does not correspond to any optical feature or star. The knot may be unrelated to the galaxy. The $(M 2-V)$ color profile is almost constant around $(M 2-V) \sim 5.3$ (Fig. 17).

IC 2006. The galaxy is classified E1 both in the RSA and Buta et al. (2015), and it is lenticular with an outer ring (RLA.-..) in the RC3. The $B$-band deep surface photometry by Schweizer et al. (1989) did reveal a faint ring. These authors suggested, however, that the entire profile, including the ring, which corresponds to $1-2 \%$ of the integrated $B$-band luminosity, is well approximated by a $r^{1 / 4}$ law, which means that the galaxy is a bona fide elliptical. Schweizer et al. (1989) found that the ring overlaps with an HI counter-rotating ring at a radius of about $150^{\prime \prime}$, inclined about $37.5^{\circ} \pm 2^{\circ}$ with respect to the plane of the sky.

In our images the outer ring starts to be recognizable in the $U$ band and becomes more prominent toward $W 2$ (Fig. 14, top panel). We measure the semi-major and minor axes of the bright UV emission, $a=151^{\prime \prime}$, and $b=129^{\prime \prime}$, and the $\mathrm{PA}=40^{\circ} \pm 2^{\circ}$. 
R. Rampazzo et al.: Investigating ETGs evolution with a multiwavelength approach. II.
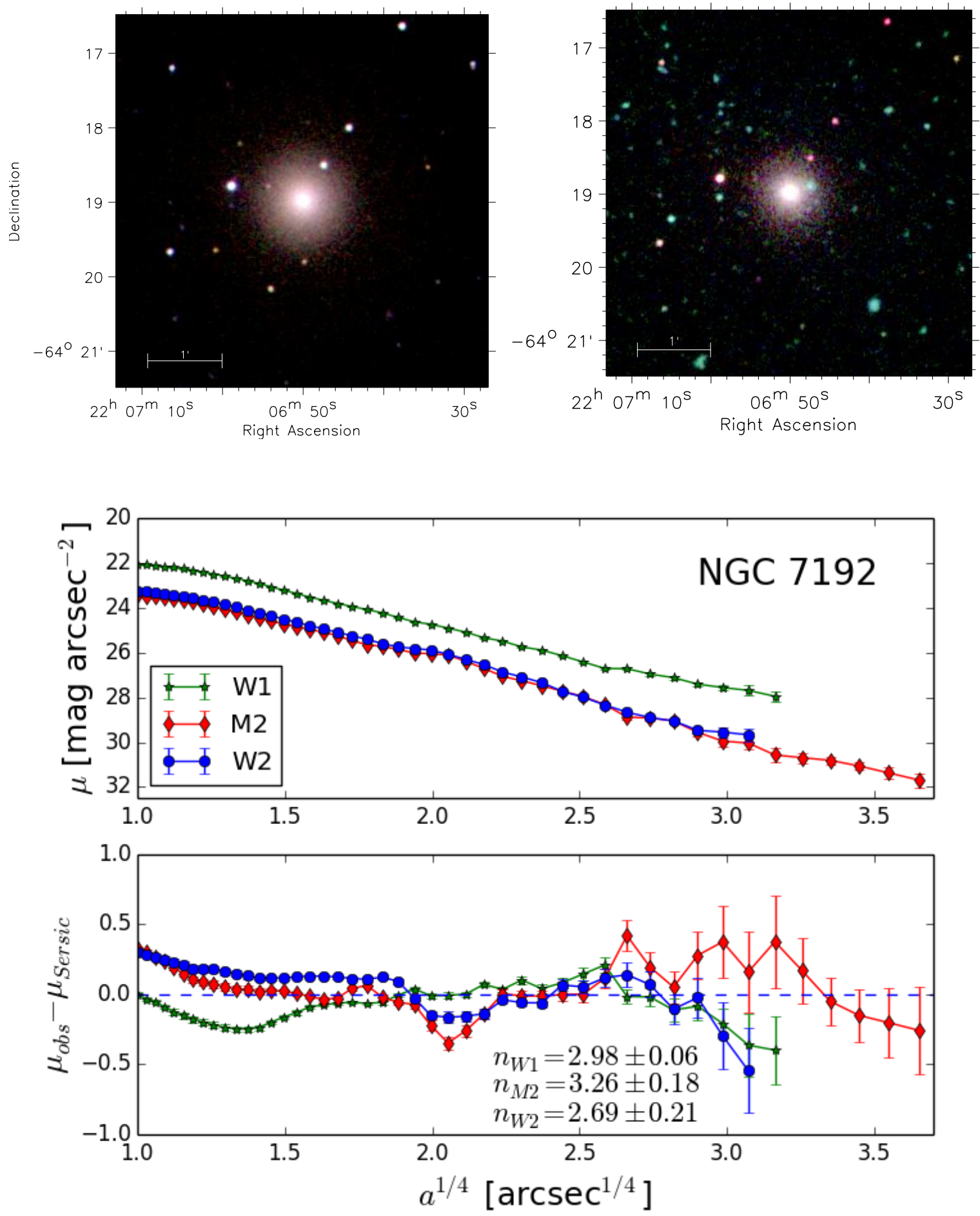

Fig. 13. As in Fig. 4 for NGC 7192. The field of view is $5^{\prime} \times 5^{\prime}$.

The ring reflects on the NUV luminosity profiles, as is shown in the bottom panel of Fig. 14. In NIR-optical CGS luminosity profiles this feature is not detected. In this context, we decided to exclude the ring and to fit the Sérsic law of our NUV profiles out to $90^{\prime \prime}$. This produces $n \leq 3.3$ in the NUV bands (bottom panel of Fig. 14). Our fit with a single Sérsic law out to $90^{\prime \prime}$ in the $B, V, R$, and $I$ profiles in the CGS gives $n<2.7$ for all of them (Fig. 15) and the same values, that is, $n<2.7$ from fitting the entire profiles.
To summarize, Sérsic indices in the range $n \sim 2$ to $n \sim$ 3.3 are found from NIR-optical to NUV, as shown in Fig. 15. A somewhat higher value, $n=3.5$, has recently been obtained by Salo et al. (2015) by analyzing MIR images of IC 2006. Our analysis then suggests that the ring- or arm-like structure should lie on an disk, although the global behavior of the $a_{4} / a$ does not show clear evidence of such an underlying feature.

The $(M 2-V)$ color profile of this galaxy is constant out to $40-45^{\prime \prime}$ (Fig. 17) and becomes progressively bluer as the 

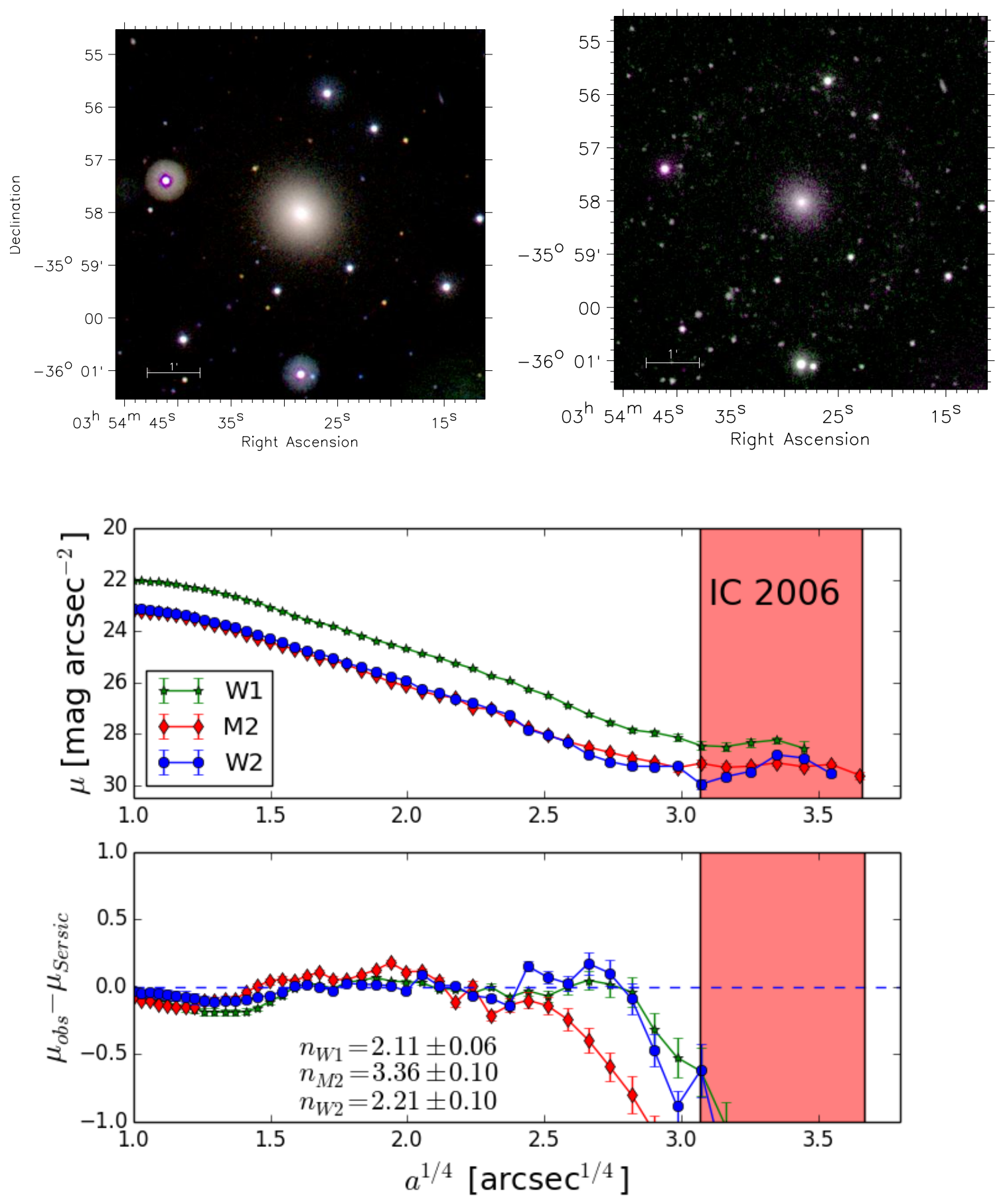

Fig. 14. As in Fig. 4 for IC 2006. The field of view is $7^{\prime} \times 7^{\prime}$. The shaded area indicates the masked region in the Sérsic law fit (see text).

galactocentric distance increases, well before $90^{\prime \prime}$, where the outer bright NUV ring emerges.

\section{Discussion}

In the introduction we asked two questions: what our NUV investigation can tell us about the history of these ETGs, and whether a common evolutionary framework exists for galaxies that show such different NUV properties. With only 11 galaxies we cannot present a coherent or exhaustive picture, in particular since we have a variety of behaviors and properties that apply sometimes to only one of the galaxies. A more general discussion will have to wait for more examples. Nonetheless, we can already see a few common features: the indication that some recent star formation can be detected in the NUV frames, which, confirmed by the Sérsic index analysis, suggests that wet accretion could be more common than previously thought even in ETGs. In the following section we connect our NUV results to the multiwavelength information available in the literature for these galaxies to explore their evolutionary framework.

\subsection{NUV galaxy structure}

Following the multiwavelength Sérsic index analysis developed in the literature from NIR to $u$ bands (La Barbera et al. 2010; Vulcani et al. 2014; Kennedy et al. 2016), we use our small sample to extend the investigation to the NUV regime. 
R. Rampazzo et al.: Investigating ETGs evolution with a multiwavelength approach. II.
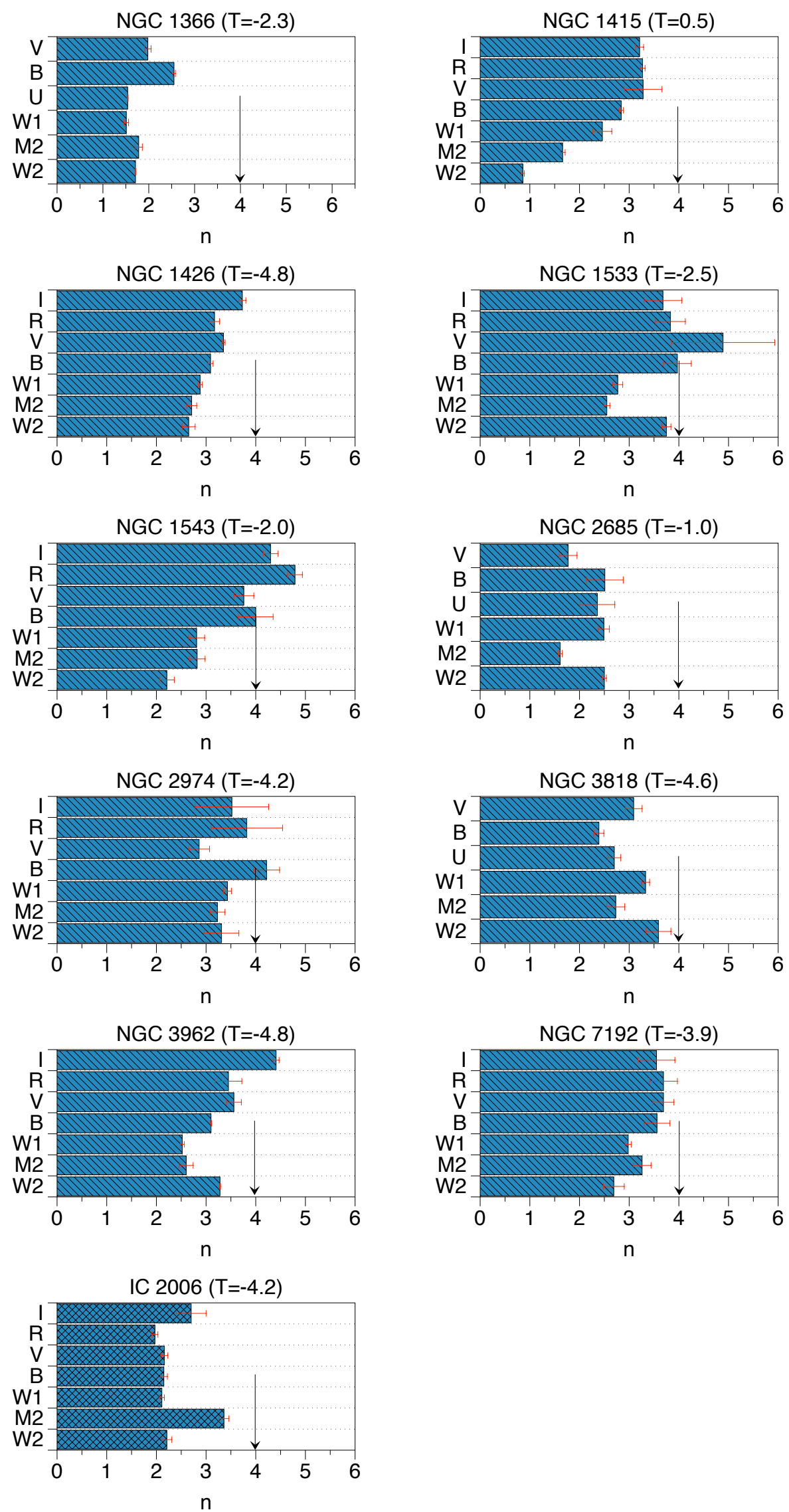

Fig. 15. Synoptic view of the Sérsic indices of the galaxies from NIR to NUV bands. NIR and optical Sérsic indices are obtained from the fit of the luminosity profiles of the CGS (Ho et al. 2011; Li et al. 2011), except for NGC 1366, NGC 2685, and NGC 3818, which are not included in that catalog. For these galaxies we used UVOT $U, B$, and $V$ bands. The morphological type, $T$, is taken from Hyperleda, as reported in Table 1 . The arrows indicate the $r^{1 / 4}$ law. 

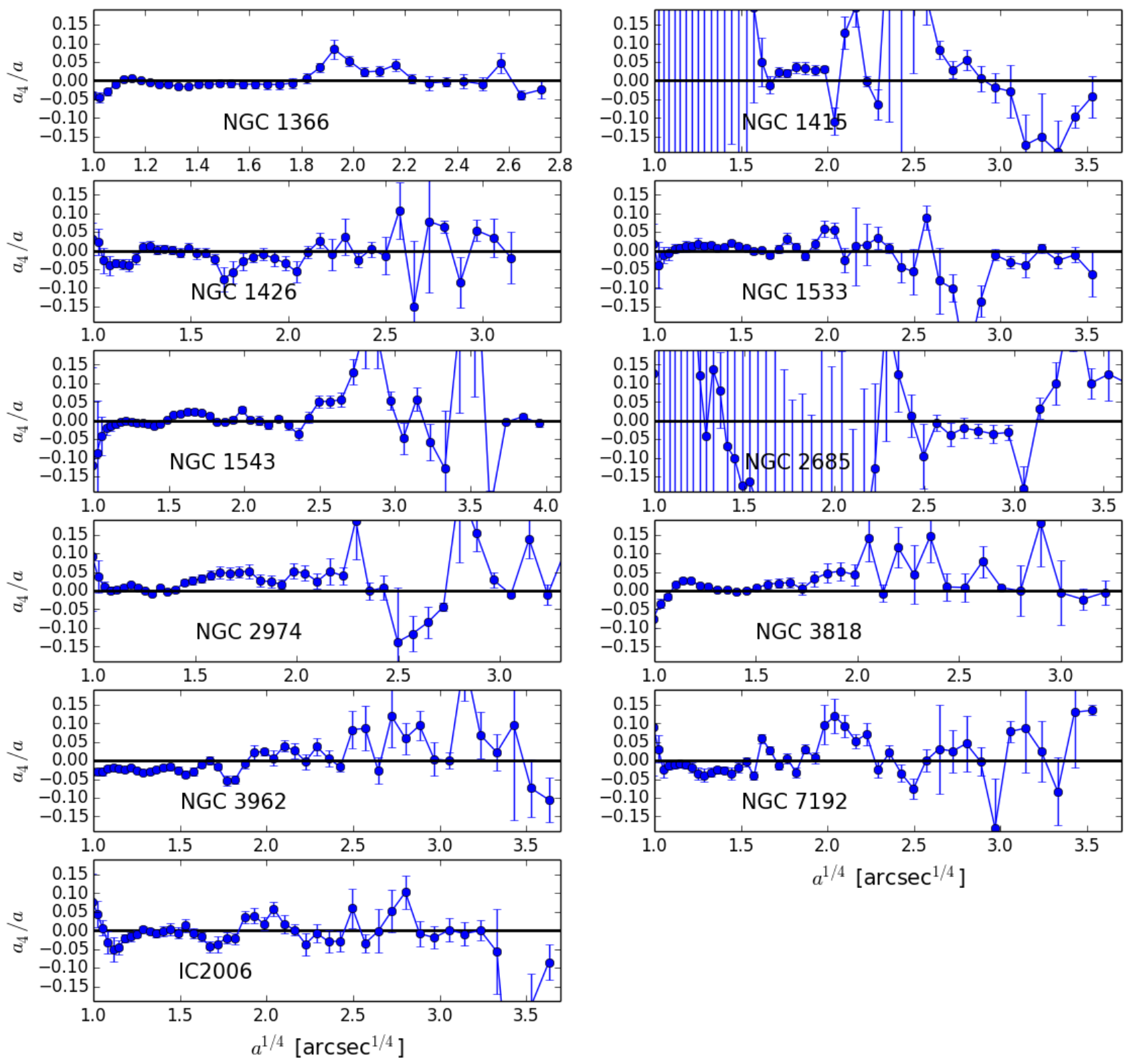

Fig. 16. Isophotal shape, $a_{4} / a$, as a function of the semi-major axis in the $M 2$ band (see also its average values in Table A.2).

Figure 15 summarizes the values of the Sérsic indices we derived, from NIR to NUV. As described in the previous section, for each galaxy the fit was performed with the aim of obtaining the description of the underlying galaxy structure in the different bands. The figure shows that most of the NUV Sérsic indexes are, within errors, in the range 2-3 or lower, whereas values corresponding to optical and NIR bands tend to be higher, $n \approx 3-4$. Based on the evidence provided by NGC 1366, where an edgeon disk is clearly visible in the NUV images and all the bands but one have $n<2$, we can interpret the low $n$ values as evidence of a disk structure in the NUV, as has previously been done at longer wavelengths. Therefore we can attribute an NUV disk to IC 2006 and NGC 2685.

When we compare the Sérsic index values within each individual galaxy, we note that variations are less extreme in Es (NGC 2974 and NGC 3818, $T<-4$ ). The widest range, consistent with the trend expected for spiral galaxies, is found in NGC 1415, with the latest morphology $(T=0.5)$. We can therefore conclude that in spite of the small size of the sample, we can reproduce the basic results found in the larger samples in optical and NIR bands: Es, differently from later types, do not show a significant variation in the Sérsic indices with wavelengths (La Barbera et al. 2010; Vulcani et al. 2014; Kennedy et al. 2016). However, with our extension in the NUV realm, Sérsic indices suggest a disk component in ETGs, which is most likely connected to a recent accretion history and a recent star formation activity.

The question is whether the color profiles indicate a disk with a bluer color than the bulge. Figure 17 shows large variation in the $(M 2-V)$ color profiles of our sample. Four galaxies, namely NGC 1426, NGC 3818, NGC 3962, and NGC 7192, have nearly constant colors. For them there is no evidence of a disk that would cause the profile to be bluer with increasing radius. Mazzei et al. (in prep.) will show by simulating our ETGs that the color of the younger galaxy populations becomes as red as $(M 2-V) \approx 5.5$ in a few $10^{8}$ yr. This behavior, that is, a global reddening of the galaxy with age, has been found in other bands by Kennedy et al. (2016). These authors found that the bulge and 
R. Rampazzo et al.: Investigating ETGs evolution with a multiwavelength approach. II.
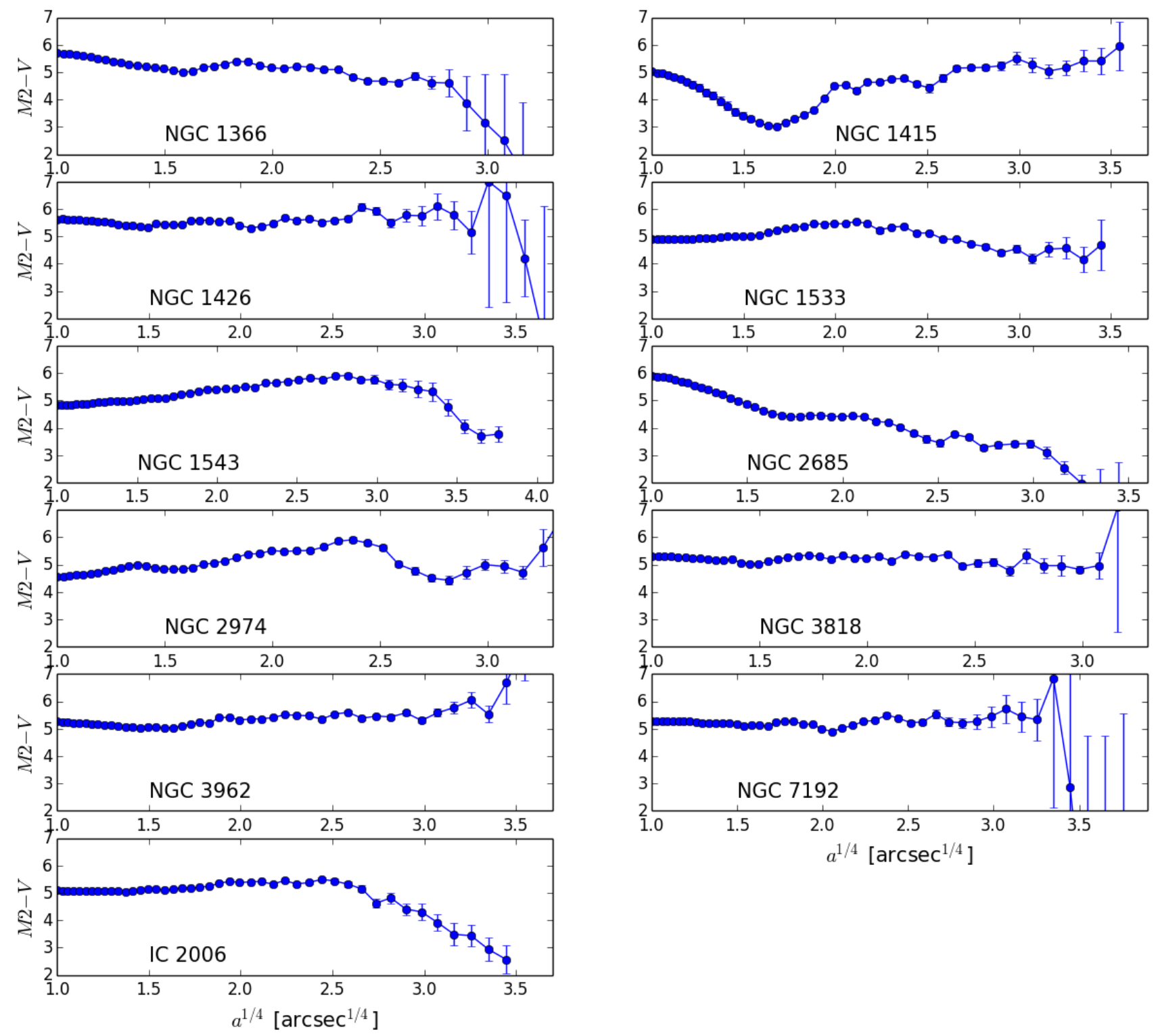

Fig. 17. $(M 2-V)$ color profiles of our ETGs sample. Profiles are corrected for the galactic extinction following Roming et al. (2009).

disk both become redder with redder total color. We note in addition that the isophotal shape (Fig. 16) suggests a disky appearance at the outskirts of both NGC 3818 and NGC 3962.

We note that the color of NGC 1366, NGC 1533, NGC 1543, NGC 2974, and IC 2006 becomes blue at large radii. The color profile of NGC 1415 is bluer in the intermediate region than it is in the outskirts, very likely as a result of dust in the outskirts. The color profile becomes nearly monotonically bluer with radius only in NGC 2685.

In summary, although the Sérsic analysis of NUV luminosity profiles with $n \approx 2-3$ suggests the presence of an underlying NUV disk independently of the morphological class and the presence of ring- or arm-like structures, the behavior of the $(M 2-V)$ color shows a variety of behaviors. Indeed, the color provides less compelling evidence since it can vary rapidly.

\subsection{Wet evolution scenario}

The presence of a disk highlighted by our NUV analysis requires a dissipative origin, or in other words, a recent wet galaxy evolution scenario. In this section we wonder whether this scenario is supported by other observations and what are the drivers of this evolution. To address these questions, we investigated SF and kinematical properties that distinguish ETGs with an unperturbed morphology (NGC 1366, NGC 1426, NGC 3818, NGC 3962, and NGC 7192) from those with ring- or armlike structures (NGC 1415, NGC 1533, NGC 1543, NGC 2685, NGC 2974, and IC 2006).

Our sample includes several signatures of star formation events even in galaxies with old or relatively old luminosityweighted nuclear ages (e.g., $r_{\mathrm{e}} / 8$ ) (Annibali et al. 2007). Amblard et al. (2014) reported a difference of a factor $\approx 55$ between the star formation rate (SFR) of NGC 2685, a polar ring galaxy with the highest SFR in our sample $(\log S F R=$ $\left.-0.36 \pm 0.41 M_{\odot} \mathrm{yr}^{-1}\right)$, and that of the nearly unperturbed galaxy NGC $1426\left(\log S F R=-2.08 \pm 0.15 M_{\odot} \mathrm{yr}^{-1}\right)$. Spitzer-IRS observations (Rampazzo et al. 2013) revealed that NGC 1533, NGC 2685, NGC 2974, and NGC 3962 show nuclear PAHs, tracers of some star formation events in the past 1-2.5 Gyr (see, e.g., Vega et al. 2010, and references therein). 
Concerning evolutionary drivers, there is a crescendo of signatures from unperturbed morphologies to those characterized by NUV ring- or arm-like structures. This supports a wet accretion scenario in our ETGs. First of all, the fundamental ingredient for a wet evolution, the neutral gas, is still abundant. Many of our ETGs own significant HI gas reservoirs (see Col. 8 in Table 1) that can feed star formation episodes. In addition, kinematical peculiarities point toward interaction or accretion events.

Of the ETGs with an unperturbed morphology, NGC 1426 and NGC 3818 do not have indication of accretion episodes. Annibali et al. (2010) reported that emission lines in the optical are weak in NGC 3818 and absent from NGC 1426, which together with the class 0 of their MIR spectra (Rampazzo et al. 2013) supports the passively evolving nature of the stellar populations in their nuclear region. Moreover, Annibali et al. (2007) reported an old luminosity-weighted age for NGC 1426 (9.0 \pm $2.5 \mathrm{Gyr})$ and for NGC $3818(8.8 \pm 1.2 \mathrm{Gyr})$ from line-strength indices analysis.

In the remaining ETGs with an unperturbed morphology, namely NGC 1366, NGC 3962, and NGC 7192, signatures of accretion are found. In these galaxies past star formation episodes may have rejuvenated the nucleus (Annibali et al. 2007; Rampazzo et al. 2013).

The nucleus of NGC 1366 has a relatively young luminosity-weighted age of $5.9 \pm 1.0 \mathrm{Gyr}$ within $r_{\mathrm{e}} / 8$ found by Annibali et al. (2010). Morelli et al. (2008) found that NGC 1366 hosts a nuclear kinematically decoupled component that is younger than the host bulge. To explain the properties of the counter-rotating component, Morelli et al. (2008) suggested that enriched material has recently been acquired through interaction or minor merging. NGC 7192 also has a relatively young nucleus $(5.7 \pm 2.0$ Gyr) (Annibali et al. 2010). Tal et al. (2009) detected a shell system around this galaxy, which is witness to its past accretion history. Carollo \& Danziger (1994) showed that stars in the innermost $8^{\prime \prime}$ region counter-rotate with respect to stars at larger radii.

NGC 3962 is a particularly intriguing case. The nuclear luminosity-weighted age of its stellar populations is $10.0 \pm$ 1.2 Gyr (Annibali et al. 2007). However, the nucleus hosts a radio source (Birkinshaw \& Davies 1985; Brown et al. 2011) and recent star formation, since there are PAHs and emission lines (MIR spectral class 2, Rampazzo et al. 2013). In addition, the arm-like structure detected by Buson (1993) in $\mathrm{H} \alpha+[\mathrm{NII}]$ supports the presence of a recent or ongoing star formation episode (Amblard et al. 2014, reported a $\log S F R=-1.32 \pm$ $\left.0.20 M_{\odot} \mathrm{yr}^{-1}\right)$. The NUV and CGS optical luminosity profiles of NGC 3962 show a sudden change in slope at $\approx 35^{\prime \prime}$. This behavior is remarkably different from the luminosity profiles of the other objects in our sample (see Sect. 4.4). Two distinct subsystems are also detected by Zeilinger et al. (1996): an inner gaseous disk hosting an old stellar bulge, and an arm-like structure. The inner disk shows regular kinematics with a major axis near PA $=70^{\circ}$, an inclination of about $45^{\circ}$, and a radius of about $5^{\prime \prime}$, which is at the limit of or better than the GALEX resolution. The brighter arm-like feature extends about $20^{\prime \prime}$, which corresponds to $3 \mathrm{kpc}$ (Table 1). The $\mathrm{H} \alpha+[\mathrm{NII}]$ emission (Zeilinger et al. 1996), however, does not extend out to the region where the change in slope of the NUV luminosity profile occurs. Neither our UVOT-NUV study nor GALEX observations (Marino et al. 2011c) show NUV features associated with the $\mathrm{H} \alpha$ emission.

Signatures of wet accretion or merging episodes are more evident in ETGs with ring- or arm-like structures.
There are no direct signatures of accretion in NGC 1415. The PA of the RL, well visible in our NUV images (see Fig. 5 top panels), differs from that of the r'l, as reported by Comerón et al. (2014). These authors found that the decoupling between the position angles of the inner and outer rings is quite normal among ringed galaxies. However, NGC 1415 is located in the FornaxEridanus cloud (Table 1, Col. 9). Since Eridanus is a loose irregular group with low velocity dispersion that is composed of 31 members, many of which are spirals (Brough et al. 2006), we cannot discard the hypothesis that the decoupling observed in NGC 1415 may be an effect of the interactions within the group.

NGC 2685, a polar-ring galaxy with dynamically decoupled gaseous and stellar components, is the most striking example for evidence of wet accretion. The outer stars rotate on a polar orbit about a central lenticular galaxy (Whitmore et al. 1990). Ulrich (1975) detected an ionized gas component that rotates fast perpendicular to the galaxy major axis (Schechter \& Gunn 1978). The kinematic separation of the two systems is also visible in $\mathrm{HI}$ (Józsa et al. 2009, and references therein).

NGC 2974, imaged in $\mathrm{H} \alpha$, presents some peripheral fainter filaments (Buson et al. 1993) and an ionized disk (Demoulin-Ulrich et al. 1984; Goudfrooij 1994) that is misaligned with respect to the stellar isophotes by $\approx 20^{\circ}$ (Pizzella et al. 1997). NGC 2974 also has an HI disk (Kim et al. 1988) with the same rotation axis as the stars. Kilborn et al. (2009) revealed HI emission in both NGC 1533 and NGC 1543 (their Fig. 4). A detailed map of the very extended HI distribution around NGC 1533 has been provided by Werk et al. (2010). IC 2006 has an HI ring corresponding to the NUV emission, as reported above (see Schweizer et al. 1989). The HI is counterrotating with respect to the stars in the central body of the galaxy. The two peaks of the stellar velocity dispersion map derived by Scott et al. (2014) with the WiFes spectrograph can be interpreted as a signature of a second counter-rotating component in the inner part of the stellar velocity field.

Our team has explored the formation mechanism and the evolution of ETGs in two nearby groups (Mazzei et al. 2014a) and of some ETGs with ring- or arm-like structures and has simulated in particular NGC 1533. We used smooth particle hydrodynamic simulations with chemo-photometric implementation starting from halos of gas and dark matter (Mazzei et al. 2014 b) to derive the dynamical and morphological evolution as well as the spectral energy distribution (SED) that extends over four orders of magnitude in wavelength at each time. In Fig. 18 the UVOT integrated magnitudes of NGC 1533, here derived, are overplotted (green open circles) on the predicted galaxy SED (red solid line). The new data agree well with the prediction by Mazzei et al. (2014b) and are consistent with their results for the evolutionary scenario of this galaxy, that is, 13.7 Gyr old according to the simulation, which suggests that NGC 1533 is the product of a wet merger following a 2:1 head-on collision. The UV ring, a transient feature in this galaxy evolution, appears when the galaxy is $\approx 8 \mathrm{Gyr}$ old, in other words, in the latter stages of the merger episode.

We conclude that multiwavelength, kinematical, and spectroscopic investigations together indicate that interaction, accretion, or merging episodes have characterized the recent history of many of our galaxies. Many of these episodes left signatures of recent star formation, either in the galaxy nuclei and/or in the outskirts. The star formation episodes give evidence that accretions or mergers have been wet, meaning that dissipation is the mechanism at the origin of the underlying disk structure that is revealed by our analysis of NUV luminosity profiles. 


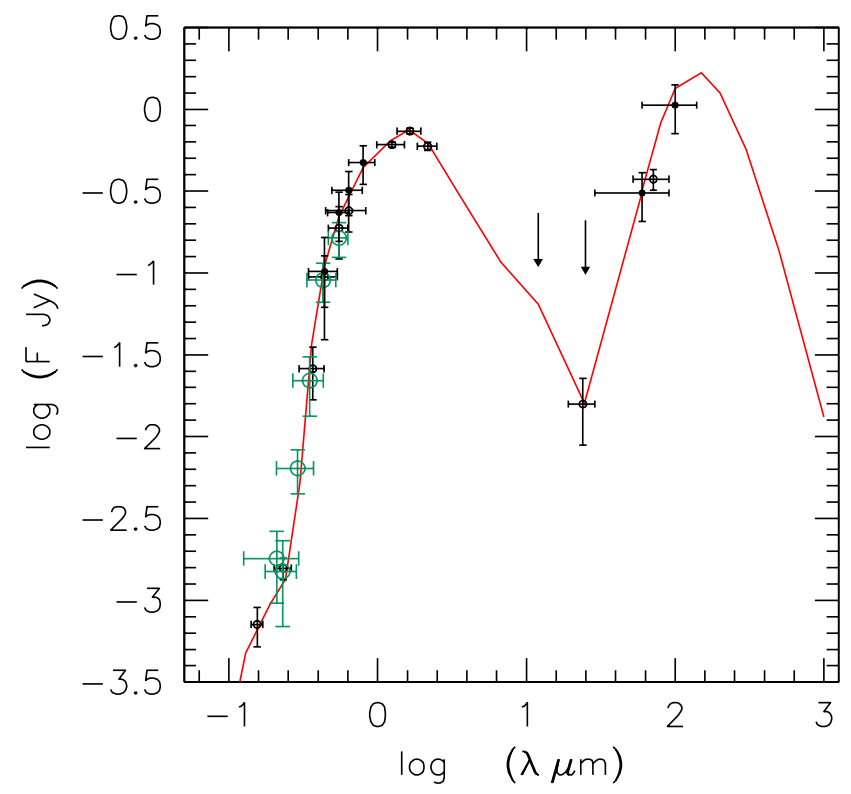

Fig. 18. SED of NGC 1533 with UVOT-NUV data (green open circles). The red solid line is the predicted SED, and black open circles and upper limits are observed data as in Mazzei et al. (2014b).

\section{Summary and conclusion}

We presented NUV surface photometry of 11 ETGs observed with Swift-UVOT. The X-ray properties of these galaxies have been presented in Paper I.

We derive their integrated magnitudes and luminosity profiles in the $W 2, M 2$, and $W 1$ NUV bands and in the optical bands.

We found a variety of behaviors, ranging from the expected featureless NUV images in about half of the galaxies we examined (NGC 1366, NGC 1426, NGC 3818, NGC 3962, and NGC 7192) to ring- or arm-like structures and evidence of a polar ring (NGC 1415, NGC 1533, NGC 1543, NGC 2974, IC 2006, and NGC 2685).

We performed a homogeneous Sérsic analysis of both our NUV and optical data from Swift and $B, V, R, I$ luminosity profiles from the CGS and report the following results. For most of our ETGs without and with ring- or arm-like structures, regardless of the optical classification, Sérsic indices in NUV are on the average in the range $n \approx 2-3$, suggesting the presence of an underlying disk. When we exclude disk galaxies (e.g., NGC 1366), the Sérsic indices from optical profiles are in general larger than those in NUV. On the average, they are in the range $n \approx 3-4$.

The $(M 2-V)$ color profiles clearly show that the ringor arm-like structures are bluer than the galaxy body, suggesting that they host ongoing or recent star formation. Featureless ETGs have red colors, but in NGC 3962 and NGC 3818 the $a_{4} / a$ isophotal shape is disky in the outskirts. We recall that the color of young stellar populations turns to red $(M 2-V) \approx 5.5$ in a few $10^{8}$ years.

The values of the Sérsic indices in the NUV reveal the role of a dissipative mechanism in these ETGs, which is clearly supported by the presence of residual HI gas. NUV unperturbed systems also present scars of accretion or merging episodes that rejuvenated the galaxy nucleus, as indicated by both their luminosity-weighted nuclear ages and PAH observations. This suggests star formation episodes in the past few Gyr. Decoupled gas-stars kinematics also supports this view.
We plan to use these far-UV data sets together with the $\mathrm{X}$-ray analysis we presented in Paper I to constrain a grid of SPH simulations with chemo-photometric implementation. This will provide a self-consistent view of the evolution of our ETGs. In Fig. 18 we show how the basic information collected here of their NUV properties will help in defining the SED of NGC 1533, which has been studied in Mazzei et al. (2014b).

We further emphasize that Swift-UVOT data represent an important NUV resource for the study of ETGs, in particular for those galaxies that do not have deep GALEX images.

Acknowledgements. We thank the anonymous referee for the careful reading and helpful suggestions that improved the paper. We would like to thank Louis Ho, Zhao-Yu Li, and the CGS team for kindly providing the $B, V, R, I$ luminosity profiles derived form the Carnegie-Irvine Galaxy Survey. Paola Mazzei and Roberto Rampazzo acknowledge support from INAF through grant PRIN-2014-14 "Star formation and evolution in galactic nuclei". We acknowledge the usage of the HyperLeda database (http://leda . univ-lyon1.fr).

\section{References}

Amblard, A., Riguccini, L., Temi, P., et al. 2014, ApJ, 783, 135 Annibali, F., Bressan, A., Rampazzo, R., Zeilinger, W. W., \& Danese, L. 2007, A\&A, 463, 455

Annibali, F., Bressan, A., Rampazzo, R., et al. 2010, A\&A, 519, A40

Bender, R., Surma, P., Döbereiner, S., Möllenhoff, C., \& Madjewsky, R. 1989, A\&A, 217, 35

Birkinshaw, M., \& Davies, R. L. 1985, ApJ, 291, 32

Breeveld, A. A., Curran, P. A., Hoversten, E. A., et al. 2010, MNRAS, 406, 1687 Breeveld, A. A., Landsman, W., Holland, S. T., et al. 2011, in Gamma Ray Bursts 2010, AIP Conf. Proc., 1358, 373

Bressan, A., Panuzzo, P., Buson, L., et al. 2006, ApJ, 639, L55

Brown, M. J. I., Januzzi, B. T., Floyd, D. J. E., \& Mould, J. R. 2011, ApJ, 731, L41

Brough, S., Forbes, D. A., Kilborn, V. A., Couch, W., \& Colless, M. 2006, MNRAS, 368, 1351

Burrows, D. N., Hill, J. E., Nousek, J. A., et al. 2005, Space Sci. Rev., 120, 165 Buson, L. 1993, Mem. Soc. Astron. It., 64, 629

Buson, L., Salder, E., Zeilinger, W., et al. 1993, A\&A, 280, 409

Buta, R. J., Sheth, K., Athanassoula, E., et al. 2015, ApJS, 217, 32

Capaccioli, M., Caon, N., \& Rampazzo, R. 1990, MNRAS, 242, 24

Caon, N., Capaccioli, M., \& D'Onofrio, M. 1993, MNRAS, 265, 1013

Capaccioli, M., Piotto, G., \& Rampazzo, R. 1988, AJ, 96, 487

Comerón, S., Salo, H., Laurikainen, E., et al. 2014, A\&A, 562, A121

Carollo, M., \& Danziger, I. J. 1994, MNRAS, 270, 523

Citterio, O., Conconi, P., Ghigo, M., et al. 1994, in SPIE Conf. Ser. 2279, eds. R.

B. Hoover, \& A. B. Walker, 480

Ulrich-Demoulin, M.-H., Butcher, H. R., \& Boksenberg, A. 1984, ApJ, 285, 527 de Vaucouleurs, G. 1948, Ann. Astrophys., 11, 247

de Vaucouleurs, G., de Vaucouleurs, A., Corwin, H. G., Jr., et al. 1991, Third

Reference Catalogue of Bright Galaxies (New York, USA: Springer)

Duc, P.-A., Cuilllandre, G.-C., Karabak, E., et al. 2015, MNRAS, 446, 120

Erwin, P., Saglia, R. P., Fabricius, M., et al. 2015, MNRAS, 446, 4039

Gadotti, D. A. 2009, MNRAS, 393, 1531

Gehrels, N., Chincarini, G., Giommi, P., et al. 2004, ApJ, 611, 1005

Goudfrooij, P. 1994, Ph.D. Thesis, University of Amsterdam, The Netherlands

Governato, F., Reduzzi, L., \& Rampazzo, R. 1996, MNRAS, 261, 379

Hernández-Pérez, F., \& Bruzual, G. 2014, MNRAS, 444, 2571

Ho, L. C., Zhao-Yu, L., Barth, A. J., et al. 2011, ApJS, 197, 21

Hodges-Kluck, E., Bregman, J. N. 2014, AJ, 789, 131

Hopkins, P. F., Cox, T. J., Dutta, S. N., et al. 2099, ApJS, 181, 135

Hoversten, E. A., Gronwall, C., Vanden Berk, D. E., et al. 2011, AJ, 141, 205

Huang, S., Ho, L. C., Peng, C. Y., Li, Z.-Y., \& Barth, A. J. 2013, ApJ, 766, 47 Jedrzejewski, R. 1987, MNRAS, 226, 747

Jeong, H., Sukyoung, K., Bureau, M., et al. 2009, MNRAS, 398, 2028

Józsa, G. I. G., Oosterloo, T. A., Morganti, R., et al. 2009, A\&A, 494, 489

Kaneda, H., Honaka, T., Sakon, L., et al. 2008, ApJ, 684, 270

Karczewski, O. Ł., Barlow, M. J., Page, M. J., et al. 2013, MNRAS, 431, 2493

Kaviraj, S., Schawinski, K., Devriendt, J. E. G., et al. 2007, ApJS, 173, 619

Kennedy, R., Bamford, S. P., Häußler, B., et al. 2016, MNRAS, 460, 3458

Kilborn, V. A., Forbes, D. A., Barnes, D. G., et al. 2009, MNRAS, 400, 1962

Kim, D.-W., Guhathakurta, P., Van Gorkom, J. H., et al. 1988, ApJ 330, 684

La Berbera, F., de Cravalho, R. R., de la Rosa, I. G., et al. 2010, MNRAS, 408, 1313

Laurikainen, E., Salo, H., Buta, R., et al. 2006, AJ, 132, 2634 
Laurikainen, E., Salo, H., Buta, R., Kanpen, J. H., \& Comerón, S. 2010, MNRAS, 405, 1089

Laurikainen, E., Salo, H., Buta, R., \& Kanpen, J. H. 2011, MNRAS, 418, 1452

Li, Z.-Y., Ho, L. C., Barth, A. J., \& Peng, C. Y. 2011, ApJS, 197, 22

Longhetti, M., Bressan, A., Chiosi, C., \& Rampazzo, R. 2000, A\&A, 353, 917

Makarov, D., Prugniel, P., Terekhova, N., Courtois, H., \& Vauglin, I. 2014, A\&A, 570, A13

Marino, A., Iodice, E., Tantalo, R., et al. 2009, A\&A, 508, 1235

Marino, A., Rampazzo, R., Bianchi, L., et al. 2011a, MNRAS, 411, 311

Marino, A., Bianchi, L., Rampazzo, R., et al. 2011b, Ap\&SS, 335, 243

Marino, A., Bianchi, L., Rampazzo, R., et al. 2011c, ApJ, 736, 154

Markwardt, C. B. 2009, in Astronomical Data Analysis Software and Systems XVIII, ASP Conf. Ser., 411, 251

Mapelli, M., Rampazzo, R., \& Marino, A. 2015, A\&A, 575, A16

Martin, D. C., Fanson, J., Schiminovich, D., et al. 2005, ApJ, 619, L1

Mazzei, P., Marino, A., \& Rampazzo, R. 2014a, ApJ, 782, 53

Mazzei, P., Marino, A., Rampazzo, R., Galletta, G., \& Bettoni, D. 2014b, Adv. Space Res., 53, 950

Morelli, L., Pompei, E., Pizzella, A., et al. 2008, MNRAS, 389, 341

Nanni, A., Bressan, A., Marigo, P., \& Girardi, L. 2013, MNRAS, 434, 2390

Oke, J. B. 1974, ApJS, 27, 21

Panuzzo, P., Rampazzo, R., Bressan, A., et al. 2011, A\&A, 528, A10

Pizzella, A., Amico, P., Bertola, F., et al. 1997, A\&A, 323, 349

Poole, T. S., Breeveld, A. A., Page, M. J., et al. 2008, MNRAS, 383, 627

Rampazzo, R., Marino, A., Tantalo, R., et al. 2007, MNRAS, 381, 245

Rampazzo, R., Annibali, F., Marino, A., et al. 2011, Astrophys. Space Sci., 335, 201

Rampazzo, R., Panuzzo, P., Vega, O., et al. 2013, MNRAS, 432, 374

Roming, P.W. A., Kennedy, T. E., Mason, K. O., et al. 2005, Space Sci. Rev., 120,95

Roming, P. W. A., Koch, T. S., Oates, S. R., et al. 2009, ApJ, 690, 163
Ryan-Weber, E., Webster, R., \& Starvely-Smith, L. 2003, MNRAS, 343, 1195 Salim, S., \& Rich, R. M. 2010, ApJ, 714, L290

Salim, S., Fang, J. J., Rich, R. M., Faber, S. M., \& Thilker, D. A. 2012, ApJ, 755, 105

Salo, H., Laurikainen, E., Laine, J., et al. 2015, ApJS, 219, 4

Sandage, A. R., \& Tammann, G. 1987, A Revised Shapley Ames Catalogue of Bright Galaxies, Carnegie, Washington (RSA)

Schawinski, K., Kaviraj, S., Khochfar, S., et al. 2007, ApJS, 173, 512

Schechter, P. L., \& Gunn, J. E. 1978, AJ, 83, 1360

Schweizer, F., van Gorkom, J. H., \& Seitzer, P. 1989, ApJ, 338, 770

Scorza, C., Bender, R., Wilkelmann, C., et al. 1998, A\&AS, 131, 265

Scott, N., Davies, R. L., Houghton, R. C. W., et al. 2014, MNRAS, 441, 274

Serra, P., \& Oosterloo, T. A. 2010, MNRAS, 401, L29

Sérsic, J. L. 1968, Atlas de Galaxias Australes (Cordoba, Argentina: Observatorio Astronomico)

Stoughton C., Lupton, R. H., Bernardi, M., et al. 2002, AJ, 123, 485

Tal, T., van Dokkum, P. G., Nelan, J., \& Bezanson, R. 2009, AJ, 138, 1417

Thilker, D. A., Bianchi, L., Schiminovic, D., et al. 2010, ApJ, 714, L171

Trinchieri, G., Rampazzo, R., Mazzei, P., Marino, A., \& Wolter, A. 2015, MNRAS, 449, 3021

Tully, R. B. 1988, Nearby Galaxy Catalog (Cambridge University Press)

Tully, R. B., Rizzi, L., Shaya, E. J., et al. 2009, AJ, 138, 323

Ulrich, M.-H. 1975, PASP, 87, 965

Vega, O., Bressan, A., Panuzzo, P., et al. 2010, ApJ, 721, 1090

Vulcani, B., Bamford, S. P., Haüßler, B., et al. 2014, MNRAS, 441, 1340

Yi, S. K., Yoon, S.-J., Kaviraj, S., et al. 2005, ApJ, 619, L111

Yi, S. K., Lee, J., Sheen, Y.-K., et al. 2011, ApJS, 195, 22

Werk, J. K., Putman, M. E., Meurer, G. R., et al. 2010, AJ, 139, 279

Whitmore, B. C., Lucas, R. A., McElroy, D. B., et al. 1990, AJ, 100, 1489

Wyder, T. K., Martin, D. C., Schiminovich, D., et al. 2007, ApJS, 173, 293

Zeilinger, W. W., Pizzella, A., Amico, P., et al. 1996, A\&AS, 120, 257 
R. Rampazzo et al.: Investigating ETGs evolution with a multiwavelength approach. II.

\section{Appendix A: Additional tables}

Table A.1. Luminosity profiles in the NUV and optical UVOT bands.

\begin{tabular}{|c|c|c|c|c|c|c|}
\hline $\begin{array}{r}a \\
{[\operatorname{arcsec}]}\end{array}$ & $\begin{array}{c}\mu_{W 2} \\
{\left[\mathrm{mag} \operatorname{arcsec}^{-2}\right]}\end{array}$ & $\begin{array}{c}\mu_{M 2} \\
{\left[\mathrm{mag} \operatorname{arcsec}^{-2}\right]}\end{array}$ & $\begin{array}{c}\mu_{W 1} \\
{\left[\mathrm{mag} \mathrm{arcsec}^{-2}\right]}\end{array}$ & $\begin{array}{c}\mu_{U} \\
{\left[\mathrm{mag} \mathrm{arcsec}^{-2}\right]}\end{array}$ & $\begin{array}{c}\mu_{B} \\
{\left[\mathrm{mag} \operatorname{arcsec}^{-2}\right]}\end{array}$ & $\begin{array}{c}\mu_{V} \\
{\left[\mathrm{mag} \mathrm{arcsec}^{-2}\right]}\end{array}$ \\
\hline \multicolumn{7}{|l|}{ NGC 1366} \\
\hline 1.0 & $22.93 \pm 0.01$ & $23.25 \pm 0.01$ & $21.51 \pm 0.01$ & $19.69 \pm 0.01$ & $18.33 \pm 0.01$ & $17.44 \pm 0.01$ \\
\hline 1.1 & $22.95 \pm 0.01$ & $23.27 \pm 0.01$ & $21.54 \pm 0.01$ & $19.72 \pm 0.01$ & $18.37 \pm 0.01$ & $17.49 \pm 0.01$ \\
\hline 1.3 & $22.99 \pm 0.01$ & $23.30 \pm 0.01$ & $21.58 \pm 0.01$ & $19.76 \pm 0.01$ & $18.41 \pm 0.01$ & $17.53 \pm 0.01$ \\
\hline 1.4 & $23.04 \pm 0.01$ & $23.34 \pm 0.01$ & $21.62 \pm 0.01$ & $19.81 \pm 0.01$ & $18.48 \pm 0.01$ & $17.60 \pm 0.01$ \\
\hline 1.6 & $23.10 \pm 0.01$ & $23.39 \pm 0.01$ & $21.66 \pm 0.01$ & $19.87 \pm 0.01$ & $18.56 \pm 0.01$ & $17.69 \pm 0.01$ \\
\hline 1.8 & $23.16 \pm 0.01$ & $23.43 \pm 0.01$ & $21.73 \pm 0.01$ & $19.95 \pm 0.01$ & $18.65 \pm 0.01$ & $17.78 \pm 0.01$ \\
\hline 2.0 & $23.23 \pm 0.01$ & $23.49 \pm 0.01$ & $21.82 \pm 0.01$ & $20.05 \pm 0.01$ & $18.76 \pm 0.01$ & $17.90 \pm 0.01$ \\
\hline 2.2 & $23.34 \pm 0.01$ & $23.61 \pm 0.01$ & $21.97 \pm 0.01$ & $20.15 \pm 0.01$ & $18.90 \pm 0.01$ & $18.03 \pm 0.01$ \\
\hline 2.5 & $23.46 \pm 0.01$ & $23.74 \pm 0.01$ & $22.12 \pm 0.01$ & $20.29 \pm 0.01$ & $19.04 \pm 0.01$ & $18.17 \pm 0.01$ \\
\hline 2.8 & $23.58 \pm 0.01$ & $23.86 \pm 0.01$ & $22.27 \pm 0.01$ & $20.44 \pm 0.01$ & $19.22 \pm 0.01$ & $18.34 \pm 0.01$ \\
\hline 3.2 & $23.71 \pm 0.01$ & $23.99 \pm 0.01$ & $22.42 \pm 0.01$ & $20.59 \pm 0.01$ & $19.41 \pm 0.01$ & $18.54 \pm 0.01$ \\
\hline 3.5 & $23.84 \pm 0.01$ & $24.11 \pm 0.01$ & $22.57 \pm 0.01$ & $20.78 \pm 0.01$ & $19.62 \pm 0.01$ & $18.74 \pm 0.01$ \\
\hline 4.0 & $23.99 \pm 0.01$ & $24.23 \pm 0.01$ & $22.72 \pm 0.01$ & $20.95 \pm 0.01$ & $19.83 \pm 0.01$ & $18.94 \pm 0.01$ \\
\hline 4.5 & $24.16 \pm 0.01$ & $24.36 \pm 0.01$ & $22.88 \pm 0.01$ & $21.14 \pm 0.01$ & $20.05 \pm 0.01$ & $19.13 \pm 0.01$ \\
\hline 5.0 & $24.32 \pm 0.01$ & $24.48 \pm 0.01$ & $23.03 \pm 0.01$ & $21.32 \pm 0.01$ & $20.23 \pm 0.01$ & $19.31 \pm 0.01$ \\
\hline 5.6 & $24.49 \pm 0.01$ & $24.61 \pm 0.01$ & $23.18 \pm 0.01$ & $21.50 \pm 0.01$ & $20.38 \pm 0.01$ & $19.48 \pm 0.01$ \\
\hline 6.3 & $24.65 \pm 0.01$ & $24.74 \pm 0.01$ & $23.34 \pm 0.01$ & $21.65 \pm 0.01$ & $20.50 \pm 0.01$ & $19.64 \pm 0.01$ \\
\hline 7.1 & $24.79 \pm 0.01$ & $24.86 \pm 0.01$ & $23.49 \pm 0.01$ & $21.80 \pm 0.01$ & $20.63 \pm 0.01$ & $19.76 \pm 0.01$ \\
\hline 7.9 & $24.94 \pm 0.01$ & $24.97 \pm 0.01$ & $23.64 \pm 0.01$ & $21.90 \pm 0.01$ & $20.72 \pm 0.01$ & $19.83 \pm 0.01$ \\
\hline 8.9 & $25.09 \pm 0.01$ & $25.06 \pm 0.01$ & $23.79 \pm 0.01$ & $22.00 \pm 0.01$ & $20.78 \pm 0.01$ & $19.91 \pm 0.01$ \\
\hline 10.0 & $25.23 \pm 0.01$ & $25.16 \pm 0.02$ & $23.91 \pm 0.01$ & $22.10 \pm 0.01$ & $20.86 \pm 0.01$ & $20.02 \pm 0.01$ \\
\hline 11.2 & $25.34 \pm 0.01$ & $25.30 \pm 0.02$ & $24.01 \pm 0.01$ & $22.19 \pm 0.01$ & $20.93 \pm 0.01$ & $20.13 \pm 0.01$ \\
\hline 12.6 & $25.44 \pm 0.01$ & $25.50 \pm 0.02$ & $24.14 \pm 0.02$ & $22.31 \pm 0.01$ & $20.98 \pm 0.01$ & $20.22 \pm 0.01$ \\
\hline 14.1 & $25.60 \pm 0.02$ & $25.69 \pm 0.02$ & $24.30 \pm 0.02$ & $22.46 \pm 0.01$ & $21.10 \pm 0.01$ & $20.33 \pm 0.01$ \\
\hline 15.8 & $25.81 \pm 0.02$ & $25.75 \pm 0.02$ & $24.48 \pm 0.02$ & $22.63 \pm 0.01$ & $21.23 \pm 0.01$ & $20.49 \pm 0.01$ \\
\hline 17.8 & $25.99 \pm 0.02$ & $25.88 \pm 0.02$ & $24.73 \pm 0.02$ & $22.84 \pm 0.01$ & $21.41 \pm 0.01$ & $20.68 \pm 0.01$ \\
\hline 19.9 & $26.20 \pm 0.02$ & $26.22 \pm 0.02$ & $25.01 \pm 0.03$ & $23.05 \pm 0.01$ & $21.61 \pm 0.01$ & $20.89 \pm 0.01$ \\
\hline 22.4 & $26.38 \pm 0.02$ & $26.34 \pm 0.03$ & $25.25 \pm 0.03$ & $23.27 \pm 0.01$ & $21.87 \pm 0.01$ & $21.10 \pm 0.01$ \\
\hline 25.1 & $26.65 \pm 0.03$ & $26.60 \pm 0.03$ & $25.45 \pm 0.03$ & $23.51 \pm 0.01$ & $22.12 \pm 0.01$ & $21.38 \pm 0.01$ \\
\hline 28.2 & $26.84 \pm 0.03$ & $26.80 \pm 0.04$ & $25.76 \pm 0.05$ & $23.78 \pm 0.02$ & $22.43 \pm 0.02$ & $21.65 \pm 0.01$ \\
\hline 31.6 & $27.21 \pm 0.04$ & $26.99 \pm 0.04$ & $26.17 \pm 0.06$ & $24.08 \pm 0.02$ & $22.72 \pm 0.02$ & $21.97 \pm 0.02$ \\
\hline 35.5 & $27.49 \pm 0.04$ & $27.29 \pm 0.05$ & $26.55 \pm 0.07$ & $24.42 \pm 0.02$ & $23.14 \pm 0.02$ & $22.34 \pm 0.02$ \\
\hline 39.8 & $27.97 \pm 0.06$ & $27.52 \pm 0.06$ & $27.02 \pm 0.11$ & $24.79 \pm 0.03$ & $23.52 \pm 0.02$ & $22.73 \pm 0.03$ \\
\hline 44.7 & $28.39 \pm 0.09$ & $27.97 \pm 0.08$ & $27.74 \pm 0.22$ & $25.19 \pm 0.04$ & $23.99 \pm 0.03$ & $23.09 \pm 0.03$ \\
\hline 50.1 & $28.83 \pm 0.14$ & $28.43 \pm 0.12$ & $\ldots$ & $25.62 \pm 0.05$ & $24.94 \pm 0.07$ & $23.58 \pm 0.04$ \\
\hline 56.2 & $\ldots$ & $\ldots$ & $\ldots$ & $26.26 \pm 0.08$ & $\ldots$ & $24.21 \pm 0.01$ \\
\hline 63.1 & $\ldots$ & $\ldots$ & $\ldots$ & $26.99 \pm 0.16$ & $\ldots$ & $25.18 \pm 0.15$ \\
\hline
\end{tabular}

Notes. Semi-major axis (Col. 1), surface brightness and error in $W 2$ (Col. 2), $M 2$ (Col. 3) and $W 1$ (Col. 4). The $U$ (Col. 5), B (Col. 6) and $V$ (Col. 7) bands are not corrected for coincidence loss. The full table is only available at the CDS. 
A\&A 602, A97 (2017)

Table A.1. continued.

\begin{tabular}{|c|c|c|c|c|c|c|}
\hline $\begin{array}{r}a \\
\text { [arcsec] }\end{array}$ & $\begin{array}{c}\mu_{W 2} \\
{\left[\mathrm{mag} \operatorname{arcsec}^{-2}\right]}\end{array}$ & $\begin{array}{c}\mu_{M 2} \\
{\left[\mathrm{mag} \mathrm{arcsec}^{-2}\right]}\end{array}$ & $\begin{array}{c}\mu_{W 1} \\
{\left[\mathrm{mag} \operatorname{arcsec}^{-2}\right]}\end{array}$ & $\begin{array}{c}\mu_{U} \\
{\left[\mathrm{mag} \mathrm{arcsec}^{-2}\right]}\end{array}$ & $\begin{array}{c}\mu_{B} \\
{\left[\mathrm{mag} \mathrm{arcsec}^{-2}\right]}\end{array}$ & $\begin{array}{c}\mu_{V} \\
{\left[\mathrm{mag} \operatorname{arcsec}^{-2}\right]}\end{array}$ \\
\hline \multicolumn{7}{|l|}{ NGC 1415} \\
\hline 1.0 & $23.38 \pm 0.01$ & $23.35 \pm 0.01$ & $21.79 \pm 0.01$ & & & $18.19 \pm 0.02$ \\
\hline 1.1 & $23.38 \pm 0.01$ & $23.33 \pm 0.01$ & $21.79 \pm 0.01$ & & & $18.21 \pm 0.02$ \\
\hline 1.3 & $23.38 \pm 0.01$ & $23.32 \pm 0.01$ & $21.79 \pm 0.01$ & & & $18.23 \pm 0.02$ \\
\hline 1.4 & $23.38 \pm 0.01$ & $23.29 \pm 0.01$ & $21.80 \pm 0.01$ & & & $18.26 \pm 0.02$ \\
\hline 1.6 & $23.38 \pm 0.01$ & $23.26 \pm 0.01$ & $21.80 \pm 0.01$ & & & $18.31 \pm 0.02$ \\
\hline 1.8 & $23.37 \pm 0.01$ & $23.24 \pm 0.01$ & $21.80 \pm 0.01$ & & & $18.36 \pm 0.02$ \\
\hline 2.0 & $23.37 \pm 0.02$ & $23.20 \pm 0.08$ & $21.81 \pm 0.05$ & & & $18.40 \pm 0.02$ \\
\hline 2.2 & $23.35 \pm 0.02$ & $23.16 \pm 0.09$ & $21.87 \pm 0.06$ & & & $18.47 \pm 0.02$ \\
\hline 2.5 & $23.35 \pm 0.03$ & $23.10 \pm 0.11$ & $21.91 \pm 0.07$ & & & $18.53 \pm 0.02$ \\
\hline 2.8 & $23.33 \pm 0.03$ & $23.00 \pm 0.13$ & $21.99 \pm 0.06$ & & & $18.61 \pm 0.02$ \\
\hline 3.2 & $23.31 \pm 0.03$ & $22.97 \pm 0.13$ & $22.07 \pm 0.06$ & & & $18.69 \pm 0.02$ \\
\hline 3.5 & $23.30 \pm 0.03$ & $22.89 \pm 0.14$ & $22.19 \pm 0.04$ & & & $18.80 \pm 0.02$ \\
\hline 4.0 & $23.26 \pm 0.03$ & $22.81 \pm 0.14$ & $22.31 \pm 0.03$ & & & $18.90 \pm 0.02$ \\
\hline 4.5 & $23.24 \pm 0.03$ & $22.71 \pm 0.12$ & $22.45 \pm 0.02$ & & & $19.02 \pm 0.02$ \\
\hline 5.0 & $23.22 \pm 0.03$ & $22.70 \pm 0.11$ & $22.61 \pm 0.02$ & & & $19.15 \pm 0.02$ \\
\hline 5.6 & $23.19 \pm 0.03$ & $22.73 \pm 0.08$ & $22.75 \pm 0.04$ & & & $19.3 \pm 0.02$ \\
\hline 6.3 & $23.18 \pm 0.04$ & $22.78 \pm 0.06$ & $22.83 \pm 0.05$ & & & $19.48 \pm 0.02$ \\
\hline 7.1 & $23.17 \pm 0.05$ & $22.88 \pm 0.04$ & $22.84 \pm 0.06$ & & & $19.70 \pm 0.02$ \\
\hline 7.9 & $23.18 \pm 0.07$ & $23.06 \pm 0.04$ & $22.86 \pm 0.06$ & & & $19.89 \pm 0.02$ \\
\hline 8.9 & $23.20 \pm 0.08$ & $23.35 \pm 0.04$ & $22.80 \pm 0.06$ & & & $20.04 \pm 0.02$ \\
\hline 10.0 & $23.24 \pm 0.10$ & $23.63 \pm 0.03$ & $22.85 \pm 0.07$ & & & $20.21 \pm 0.02$ \\
\hline 11.2 & $23.30 \pm 0.12$ & $23.95 \pm 0.03$ & $23.02 \pm 0.07$ & & & $20.37 \pm 0.02$ \\
\hline 12.6 & $23.38 \pm 0.13$ & $24.29 \pm 0.04$ & $23.37 \pm 0.06$ & & & $20.54 \pm 0.01$ \\
\hline 14.1 & $23.50 \pm 0.14$ & $24.84 \pm 0.04$ & $23.89 \pm 0.05$ & & & $20.67 \pm 0.01$ \\
\hline 15.8 & $23.66 \pm 0.15$ & $25.43 \pm 0.03$ & $24.45 \pm 0.03$ & & & $20.80 \pm 0.01$ \\
\hline 17.8 & $23.88 \pm 0.15$ & $25.55 \pm 0.03$ & $24.74 \pm 0.02$ & & & $20.80 \pm 0.01$ \\
\hline 19.9 & $24.18 \pm 0.14$ & $25.47 \pm 0.07$ & $24.76 \pm 0.04$ & & & $21.00 \pm 0.02$ \\
\hline 22.4 & $24.50 \pm 0.1$ & $25.87 \pm 0.04$ & $24.91 \pm 0.03$ & & & $21.1 \pm 0.01$ \\
\hline 25.1 & $24.85 \pm 0.11$ & $25.93 \pm 0.03$ & $25.01 \pm 0.02$ & & & $21.16 \pm 0.02$ \\
\hline 28.2 & $25.13 \pm 0.09$ & $26.13 \pm 0.04$ & $25.17 \pm 0.02$ & & & $21.24 \pm 0.02$ \\
\hline 31.6 & $25.31 \pm 0.07$ & $26.27 \pm 0.07$ & $25.28 \pm 0.04$ & & & $21.36 \pm 0.02$ \\
\hline 35.5 & $25.37 \pm 0.05$ & $26.10 \pm 0.09$ & $25.29 \pm 0.07$ & & & $21.40 \pm 0.02$ \\
\hline 39.8 & $25.40 \pm 0.05$ & $26.01 \pm 0.13$ & $25.36 \pm 0.10$ & & & $21.45 \pm 0.02$ \\
\hline 44.7 & $25.35 \pm 0.06$ & $26.67 \pm 0.12$ & $25.89 \pm 0.07$ & & & $21.75 \pm 0.02$ \\
\hline 50.1 & $26.17 \pm 0.03$ & $27.44 \pm 0.08$ & $26.35 \pm 0.07$ & & & $22.15 \pm 0.02$ \\
\hline 56.2 & $27.28 \pm 0.03$ & $27.96 \pm 0.07$ & $26.75 \pm 0.06$ & & & $22.66 \pm 0.03$ \\
\hline 63.1 & $27.86 \pm 0.05$ & $28.43 \pm 0.08$ & $27.15 \pm 0.05$ & & & $23.22 \pm 0.04$ \\
\hline 70.8 & $28.20 \pm 0.06$ & $28.80 \pm 0.01$ & $27.43 \pm 0.06$ & & & $23.43 \pm 0.05$ \\
\hline 79.4 & $28.42 \pm 0.07$ & $29.32 \pm 0.15$ & $27.61 \pm 0.08$ & & & $23.67 \pm 0.07$ \\
\hline 89.1 & $28.78 \pm 0.10$ & $29.41 \pm 0.18$ & $27.89 \pm 0.09$ & & & $24.00 \pm 0.09$ \\
\hline 100.0 & $29.0 \pm 0.11$ & $29.27 \pm 0.15$ & $28.03 \pm 0.11$ & & & $24.09 \pm 0.09$ \\
\hline 112.2 & $29.20 \pm 0.14$ & $29.51 \pm 0.18$ & $28.22 \pm 0.13$ & & & $24.21 \pm 0.10$ \\
\hline 125.9 & $29.37 \pm 0.15$ & $29.99 \pm 0.269$ & $28.44 \pm 0.15$ & & & $24.44 \pm 0.13$ \\
\hline 141.3 & $30.20 \pm 0.23$ & $\ldots$ & $28.69 \pm 0.19$ & & & $24.68 \pm 0.15$ \\
\hline 158.5 & $\ldots$ & $\ldots$ & $28.99 \pm 0.23$ & & & $25.03 \pm 0.21$ \\
\hline
\end{tabular}


R. Rampazzo et al.: Investigating ETGs evolution with a multiwavelength approach. II.

Table A.2. The NUV structural properties of galaxies.

\begin{tabular}{|c|c|c|c|c|c|c|}
\hline Band & $\begin{array}{c}\langle\epsilon\rangle \\
{[1-b / a]}\end{array}$ & $\begin{array}{l}\langle\mathrm{PA}\rangle \\
{[\mathrm{deg}]}\end{array}$ & $\begin{array}{c}\left\langle a_{4} / a\right\rangle \\
\times 100\end{array}$ & $n$ & $\begin{array}{c}a_{(80)} \\
{[\operatorname{arcsec}]}\end{array}$ & Notes \\
\hline & & & NGC 1366 & & & \\
\hline$W 2$ & $0.40 \pm 0.03$ & $1.4 \pm 2.4$ & $-4.3 \pm 14.3$ & $1.71 \pm 0.01$ & 34.7 & (1) \\
\hline$M 2$ & $0.40 \pm 0.09$ & $2.2 \pm 1.8$ & $1.4 \pm 4.4$ & $1.78 \pm 0.08$ & 42.2 & (1) \\
\hline \multirow[t]{2}{*}{$W 1$} & $0.47 \pm 0.15$ & $179.8 \pm 3.7$ & $1.8 \pm 3.1$ & $1.51 \pm 0.05$ & 23.5 & (1) \\
\hline & & & NGC 1415 & & & \\
\hline$W 2$ & $0.60 \pm 0.17$ & $141.2 \pm 6.3$ & $-34.9 \pm 60.0$ & $2.46 \pm 0.19$ & 74.4 & (1) \\
\hline$M 2$ & $0.33 \pm 0.12$ & $143.6 \pm 24.8$ & $8.7 \pm 36.1$ & $1.66 \pm 0.05$ & 57.4 & (1) \\
\hline \multirow[t]{2}{*}{$W 1$} & $0.34 \pm 0.09$ & $137.1 \pm 19.5$ & $0.5 \pm 18.2$ & $0.85 \pm 0.05$ & 98.2 & (1) \\
\hline & & & NGC 1426 & & & \\
\hline$W 2$ & $0.30 \pm 0.05$ & $103.6 \pm 19.4$ & $0.4 \pm 10.3$ & $2.65 \pm 0.13$ & 61.7 & (1) \\
\hline$M 2$ & $0.30 \pm 0.04$ & $109.5 \pm 18.2$ & $-1.0 \pm 9.5$ & $2.71 \pm 0.10$ & 82.7 & (1) \\
\hline \multirow[t]{2}{*}{$W 1$} & $0.29 \pm 0.06$ & $117.7 \pm 34.59$ & $-0.5 \pm 5.1$ & $2.86 \pm 0.04$ & 148.5 & (1) \\
\hline & & & NGC 1533 & & & \\
\hline$W 2$ & $0.22 \pm 0.06$ & $160.2 \pm 2.5$ & $-3.3 \pm 8.0$ & $2.76 \pm 0.10$ & 85.5 & $(1)^{a}$ \\
\hline$M 2$ & $0.20 \pm 0.07$ & $174.6 \pm 12.9$ & $-1.8 \pm 7.6$ & $2.54 \pm 0.06$ & 85.5 & $(1)^{a}$ \\
\hline \multirow[t]{2}{*}{$W 1$} & $0.12 \pm 0.09$ & $130.7 \pm 51.7$ & $0.2 \pm 4.3$ & $3.74 \pm 0.09$ & 113.7 & $(1)^{a}$ \\
\hline & & & NGC 1543 & & & \\
\hline$W 2$ & $0.20 \pm 0.09$ & $120.5 \pm 40.8$ & $2.8 \pm 10.3$ & $2.81 \pm 0.16$ & 197.3 & (2) \\
\hline$M 2$ & $0.15 \pm 0.10$ & $130.7 \pm 53.5$ & $4.3 \pm 13.8$ & $2.82 \pm 0.16$ & 187.9 & (2) \\
\hline \multirow[t]{2}{*}{$W 1$} & $0.21 \pm 0.11$ & $130.4 \pm 53.2$ & $2.3 \pm 9.0$ & $2.21 \pm 0.15$ & 179.0 & (2) \\
\hline & & & NGC 2685 & & & \\
\hline$W 2$ & $0.47 \pm 0.02$ & $35.1 \pm 1.12$ & $-4.1 \pm 27.1$ & $2.50 \pm 0.04$ & 102.0 & (1) \\
\hline$M 2$ & $0.55 \pm 0.22$ & $54.6 \pm 35.6$ & $-13.7 \pm 21.3$ & $1.60 \pm 0.04$ & 86.1 & (1) \\
\hline \multirow[t]{2}{*}{$W 1$} & $0.44 \pm 0.07$ & $39.8 \pm 28.8$ & $-0.6 \pm 6.6$ & $2.49 \pm 0.11$ & 87.2 & (1) \\
\hline & & & NGC 2974 & & & \\
\hline$W 2$ & $0.38 \pm 0.06$ & $45.2 \pm 2.5$ & $-0.3 \pm 7.9$ & $3.31 \pm 0.35$ & 82.7 & $(2)^{a}$ \\
\hline$M 2$ & $0.24 \pm 0.06$ & $40.6 \pm 4.7$ & $3.2 \pm 10.4$ & $3.23 \pm 0.15$ & 78.8 & $(2)^{a}$ \\
\hline \multirow[t]{2}{*}{$W 1$} & $0.35 \pm 0.04$ & $46.5 \pm 4.1$ & $0.6 \pm 3.9$ & $3.43 \pm 0.08$ & 82.7 & $(2)^{a}$ \\
\hline & & & NGC 3818 & & & \\
\hline$W 1$ & $0.33 \pm 0.06$ & $108.0 \pm 34.1$ & $1.0 \pm 5.7$ & $3.59 \pm 0.25$ & 58.8 & (1) \\
\hline$M 2$ & $0.23 \pm 0.05$ & $108.2 \pm 27.3$ & $2.5 \pm 8.8$ & $2.73 \pm 0.18$ & 56.0 & (1) \\
\hline \multirow[t]{2}{*}{$W 2$} & $0.32 \pm 0.06$ & $101.3 \pm 32.9$ & $1.3 \pm 8.3$ & $3.33 \pm 0.20$ & 75.0 & (1) \\
\hline & & & NGC 3962 & & & \\
\hline$W 2$ & $0.24 \pm 0.07$ & $15.4 \pm 5.0$ & $-2.4 \pm 6.0$ & $3.28 \pm 0.07$ & 122.2 & (1) \\
\hline$M 2$ & $0.22 \pm 0.02$ & $8.8 \pm 2.5$ & $-2.4 \pm 1.0$ & $2.61 \pm 0.14$ & 75.0 & (1) \\
\hline \multirow[t]{2}{*}{$W 1$} & $0.18 \pm 0.04$ & $4.5 \pm 28.0$ & $-2.9 \pm 5.6$ & $2.52 \pm 0.04$ & 105.5 & (1) \\
\hline & & & NGC 7192 & & & \\
\hline$W 2$ & $0.09 \pm 0.05$ & $15.5 \pm 57.8$ & $0.6 \pm 6.5$ & $2.69 \pm 0.21$ & 50.8 & (1) \\
\hline$M 2$ & $0.08 \pm 0.06$ & $26.6 \pm 69.5$ & $1.1 \pm 9.2$ & $3.26 \pm 0.18$ & 61.7 & (1) \\
\hline \multirow[t]{2}{*}{$W 1$} & $0.06 \pm 0.03$ & $146.8 \pm 80.3$ & $0.3 \pm 6.1$ & $2.98 \pm 0.06$ & 100.5 & (1) \\
\hline & & & IC 2006 & & & \\
\hline$W 2$ & $0.08 \pm 0.03$ & $32.2 \pm 29.8$ & $-0.3 \pm 4.2$ & $2.21 \pm 0.10$ & 137.6 & (2) \\
\hline$M 2$ & $0.13 \pm 0.05$ & $177.4 \pm 60.4$ & $-2.5 \pm 10.7$ & $3.36 \pm 0.10$ & 151.4 & (2) \\
\hline$W 1$ & $0.07 \pm 0.02$ & $58.2 \pm 21.0$ & $-0.4 \pm 6.4$ & $2.11 \pm 0.06$ & 113.8 & (2) \\
\hline
\end{tabular}

Notes. The ellipticity (Col. 2), the position angle (Col. 3) and the isophotal shape, $a_{4} / a \times 100$, (Col. 4) are the mean values calculated in the same range of the luminosity profile, excluding the central $2 \times F W H M_{\mathrm{PSF}}$. The errors correspond to the standard deviation. The Sérsic index of the single fit is given in Col. 5. The semi-major axis including $80 \%$ of the integrated magnitude is given in Col. 6. Notes: The fit on optical and NIR profiles is made on the same extension as in the NUV profiles. In particular: (1) the single Sérsic law fit is performed without masking. (1) ${ }^{a}$ NGC 1533: the Sérsic fit is performed without masking the NUV profile (Fig. 7) while in BVRI we mask the region $20^{\prime \prime}-90^{\prime \prime}$ where the bar and the lens are present. (2) The Sérsic fit excludes the ring in NUV and BVRI profiles as shown in Figs. 8 and 14. (2) ${ }^{a}$ The NGC 2974 fit in the NUV is performed masking the region from $30^{\prime \prime}$ to $100^{\prime \prime}$ (Fig. 10) while in $B V R I$ no masking has been applied. See Sect. 4.4 for details about $B, V, R, I$ Sérsic fits. 